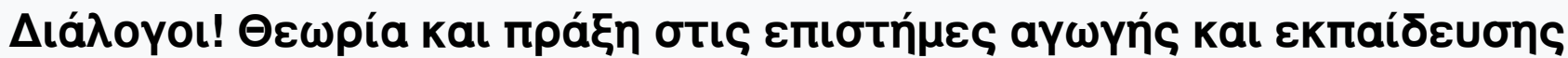

Tó 2 (2016)

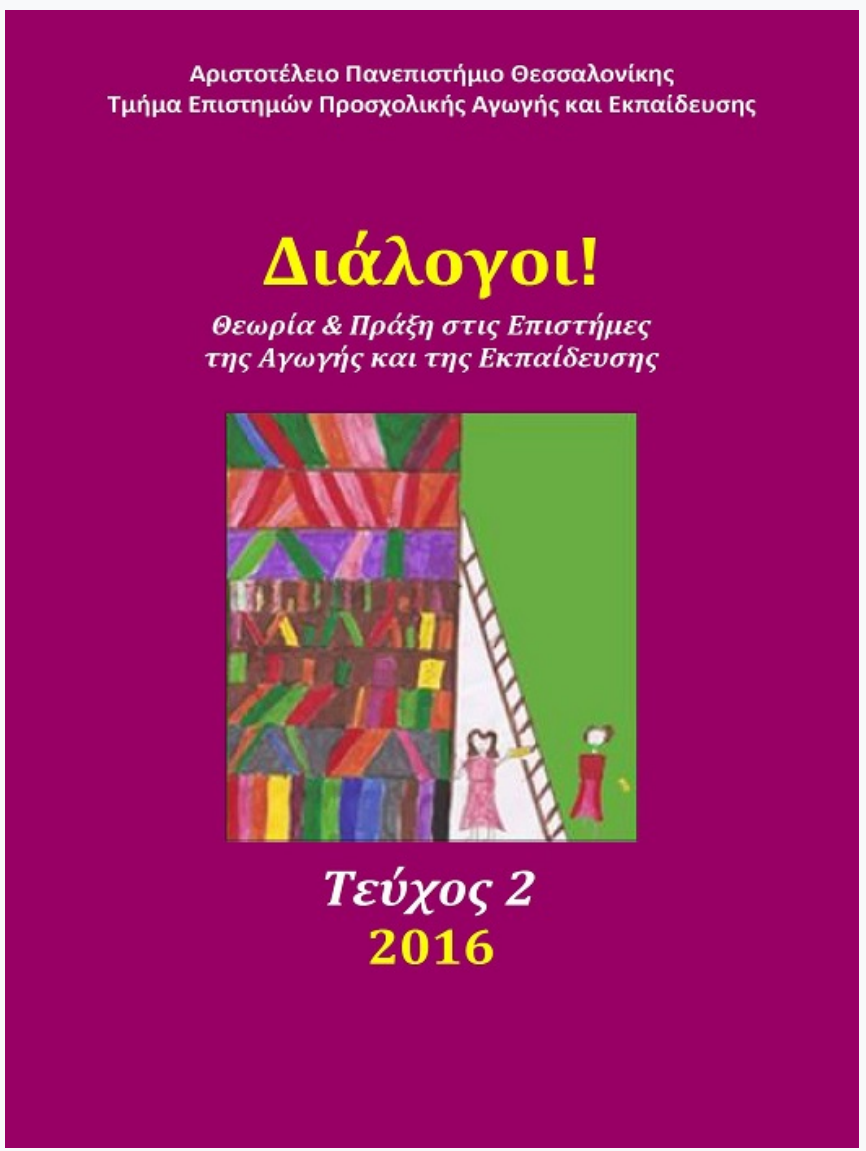

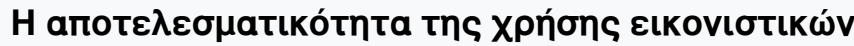

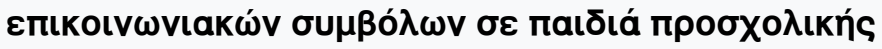

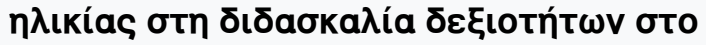
Badminton.

Athanasia Giakouvaki, Panagiotis Antoniou, Evangelos Bebetsos, Eleni Zetou

doi: $10.12681 /$ dial.10399

Copyright $\odot$ 2016, Athanasia Giakouvaki, Panagiotis Antoniou, Evangelos Bebetsos, Eleni Zetou

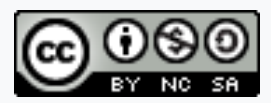

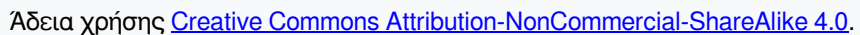

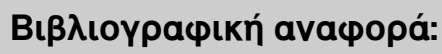

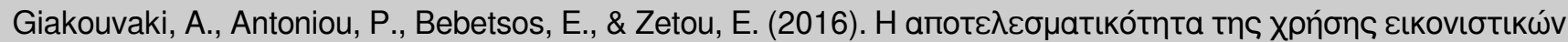

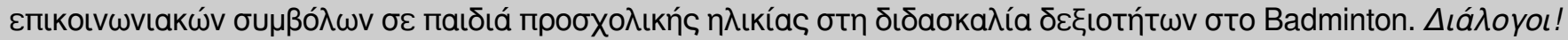

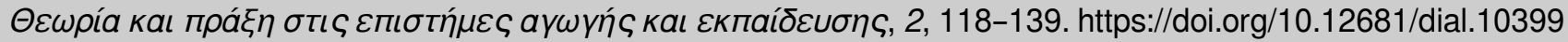




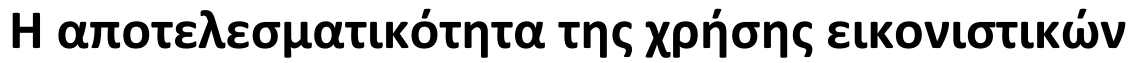

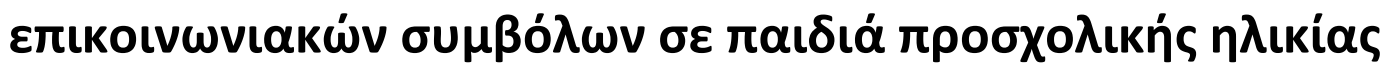

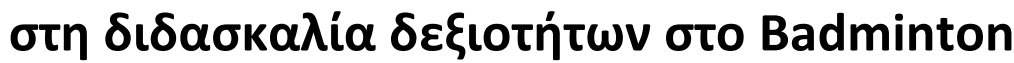

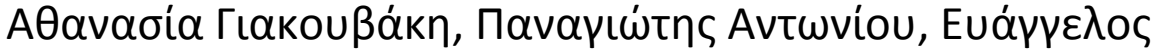

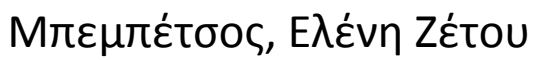

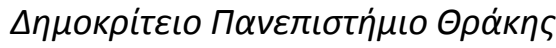

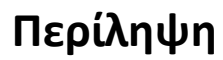

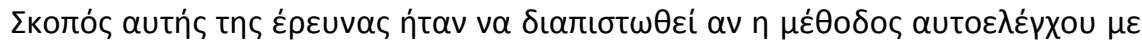

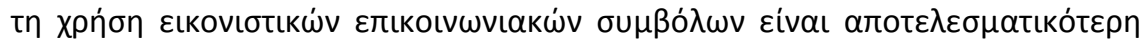

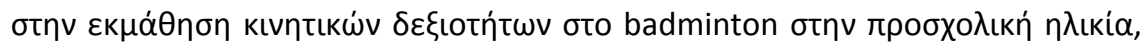

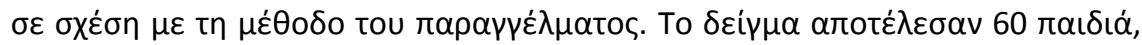

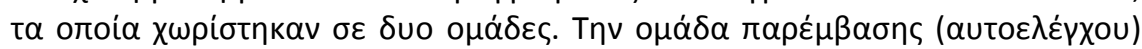

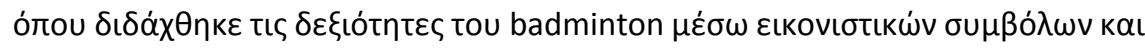

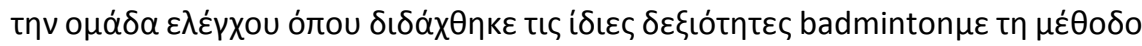

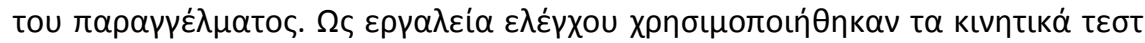
French and Stalter (Barrow \& McGee, 1979. French, 1941. Washington, 1968) kal

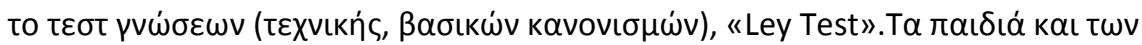

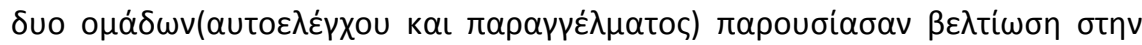

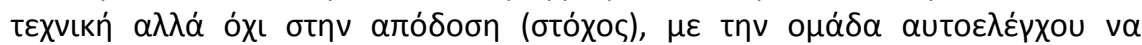

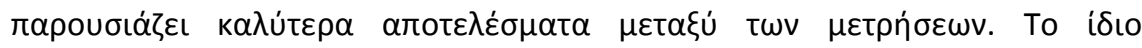

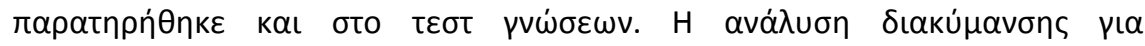

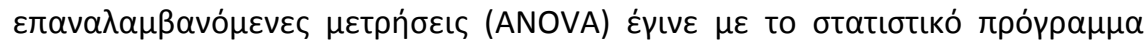

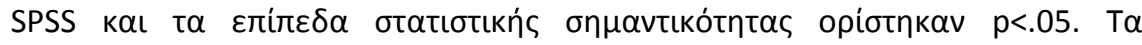
a

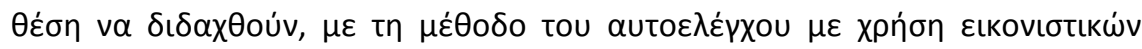

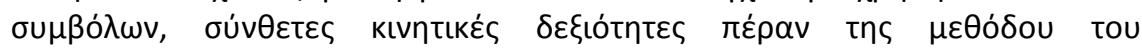

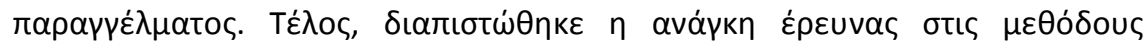

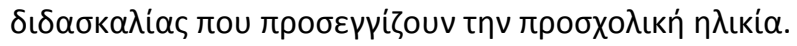

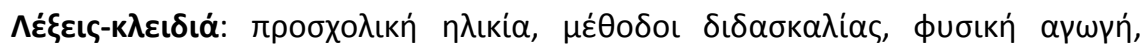

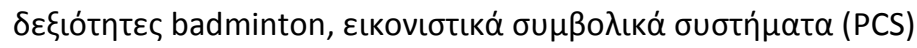

\begin{abstract}
The purpose of this study was to determine if self- check method is more effective than command method in learning badminton skills at preschool age. The sample consisted of 60 children that were divided into two groups, the experimental group (self- check) were taught badminton skills through picture communication symbols and the control group were taught the same badminton skills through the method of command. As control tools we used the French and Stalter tests (Barrow \& Mc Gee, 1979; French, 1941; Washington, 1968) and the knowledge test with technical, basic regulations and rules, ie. "Ley Test». The
\end{abstract}

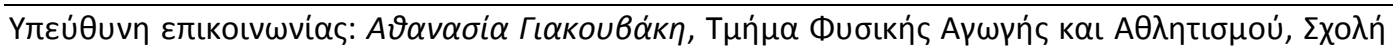

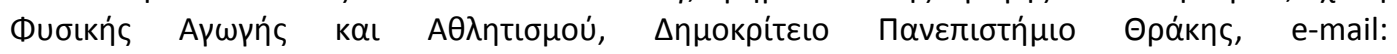
athanasiagiak@yahoo.gr 
children of both groups showed improvement in technique and not in performance (target), with the self-control group showing better results between measurements compared with the group of command. The same was observed in the knowledge test. The data analysis package SPSS was used to analyse the data and the statistical significance was accepted at $p<.05$. The conclusion of this research is that the self-check method can be used in pre-school-age children for teaching complex motor skills. It also revealed the need to research on teaching methods appropriate for children at preschool age.

Keywords: preschool age, teaching methods, physical education, skills of badminton, picture communication symbols (PCS)

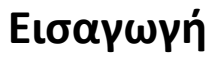

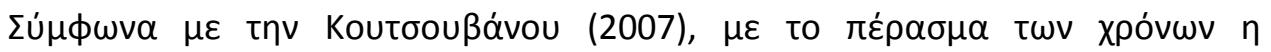

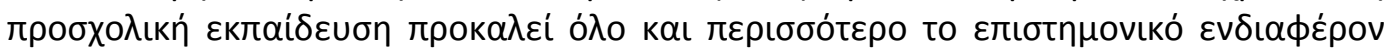

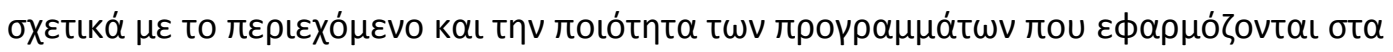

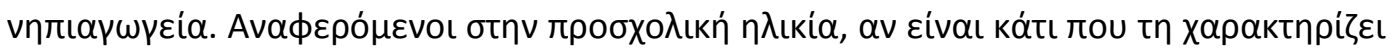

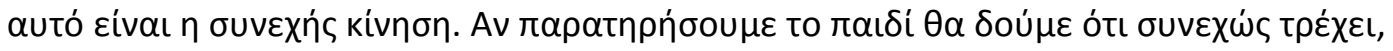

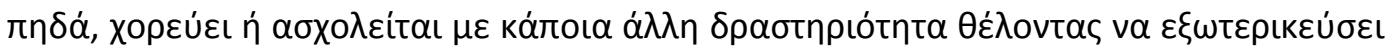

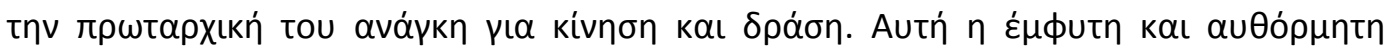

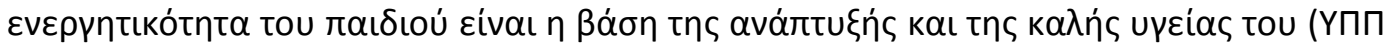

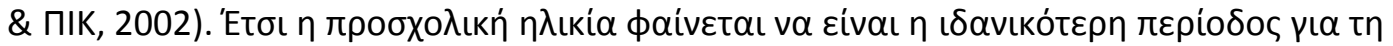

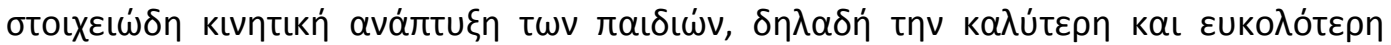

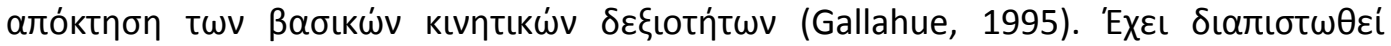

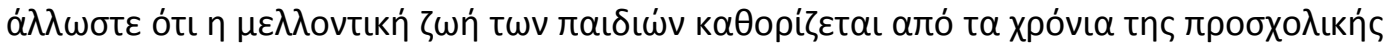

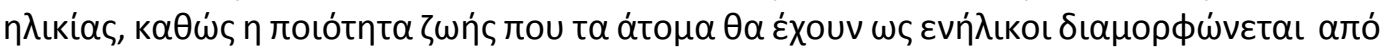

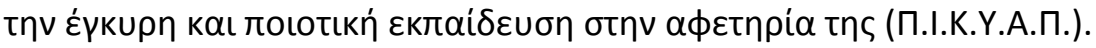

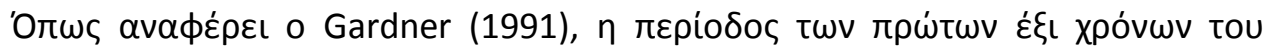

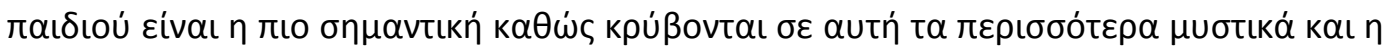

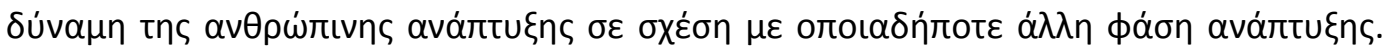

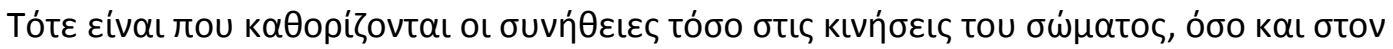

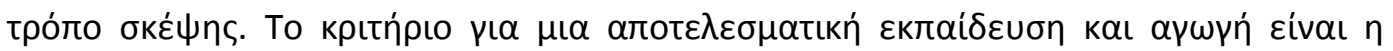

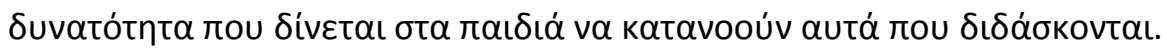

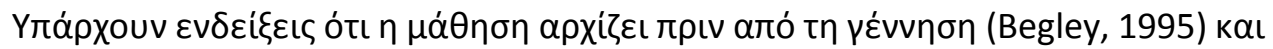

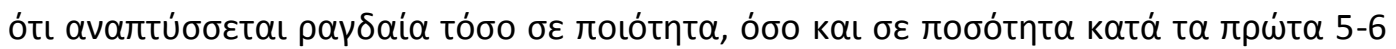

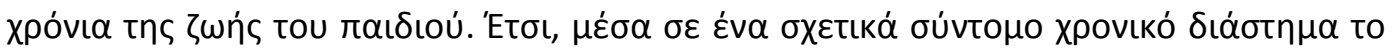

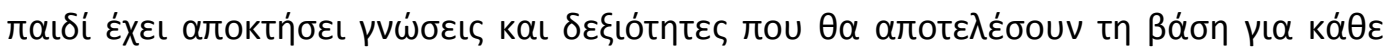

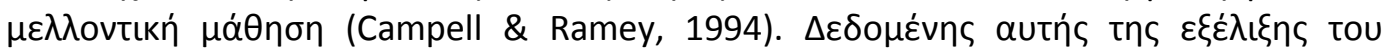

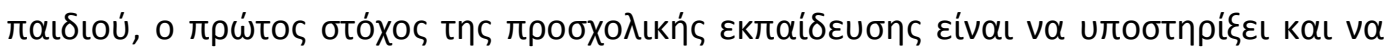

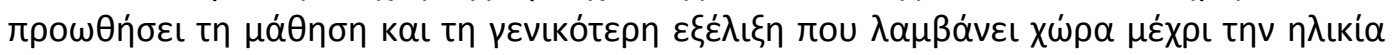

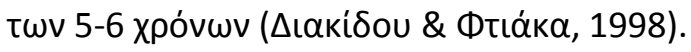

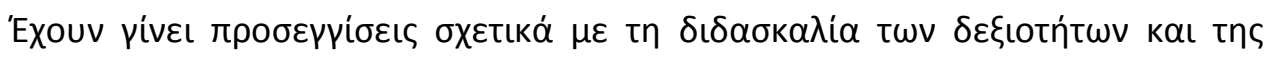

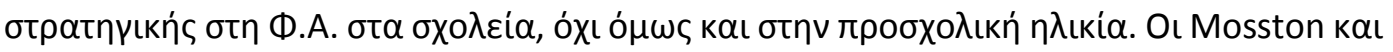

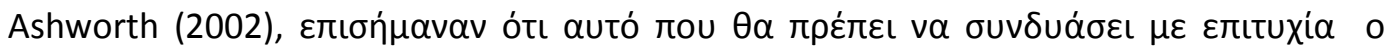

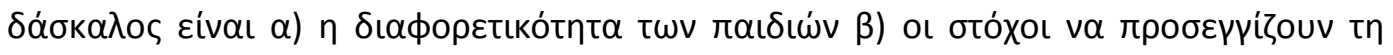

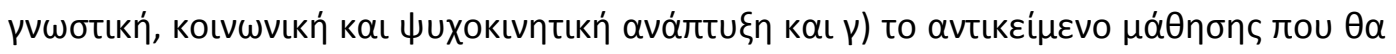




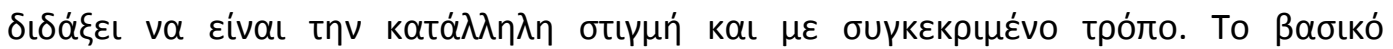

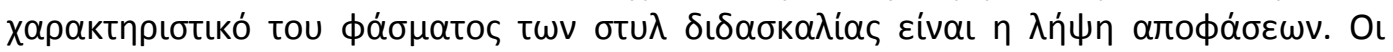

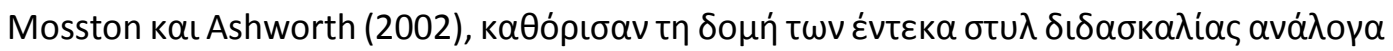

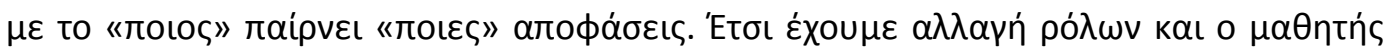

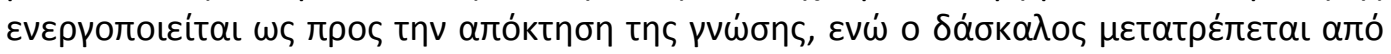

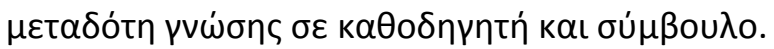

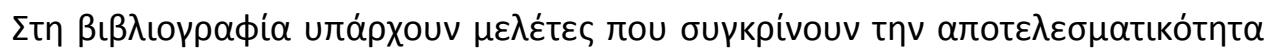

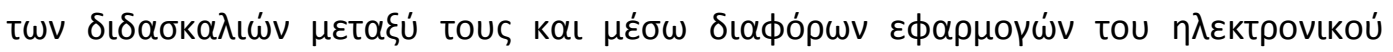

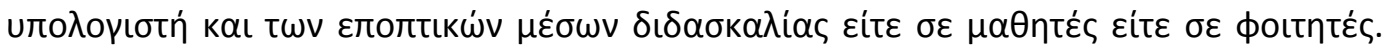

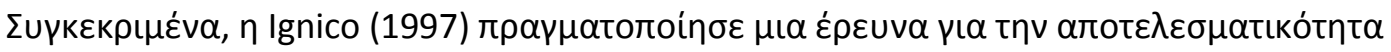

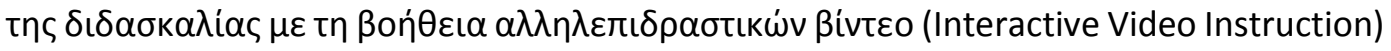

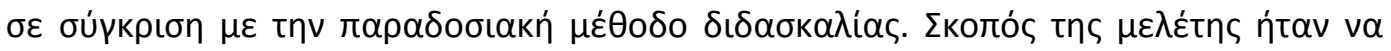

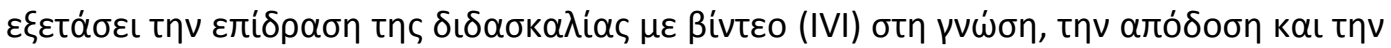

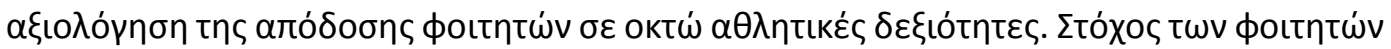

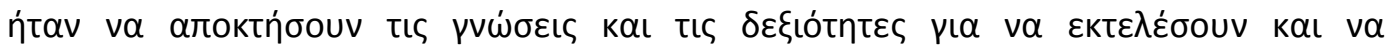

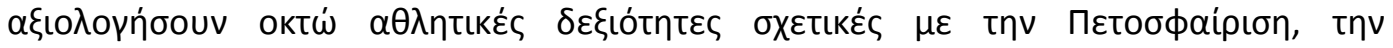

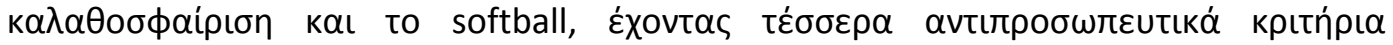

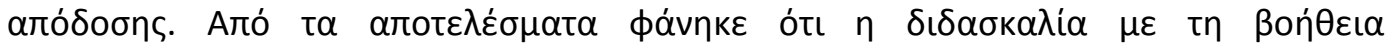

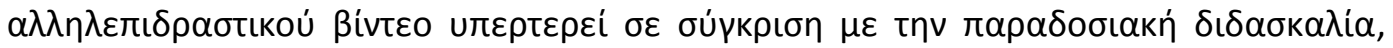

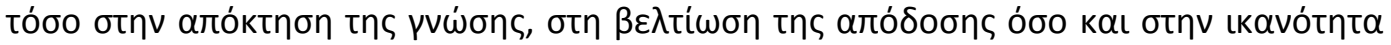

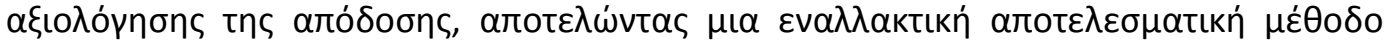
$\delta ı \delta \alpha \sigma \alpha \lambda i \alpha \varsigma$.

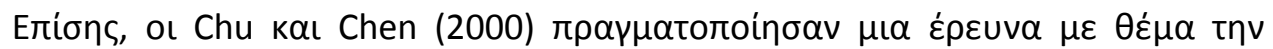

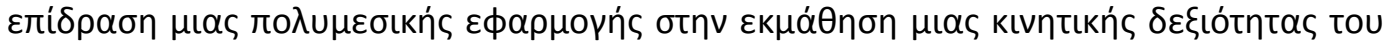

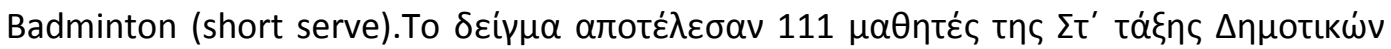

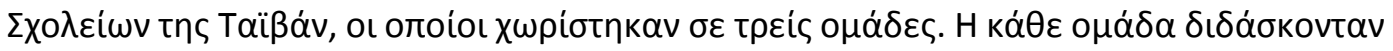

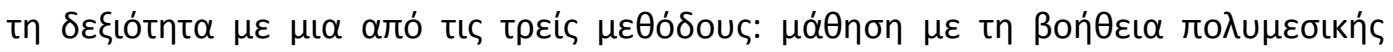

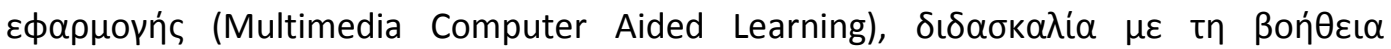

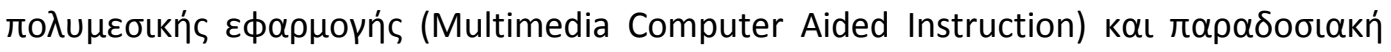

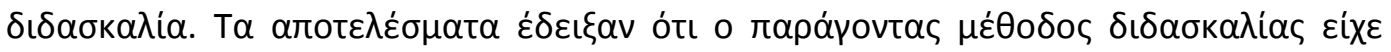

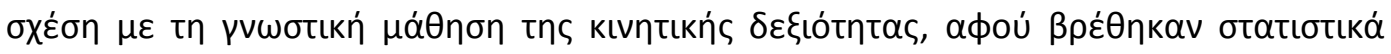

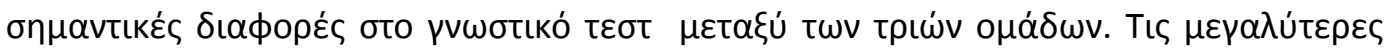

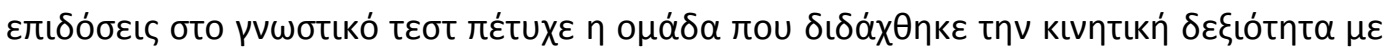

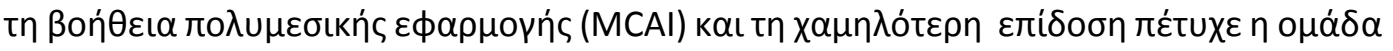

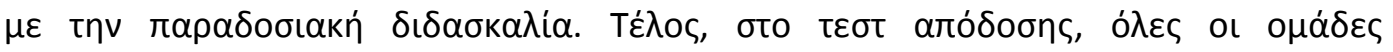

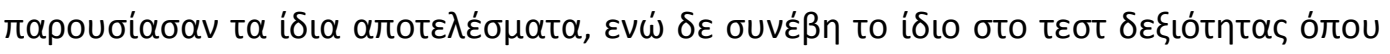

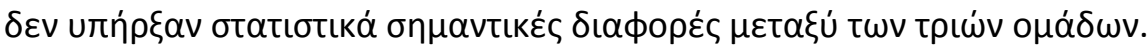

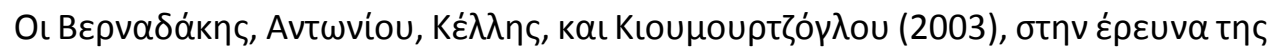

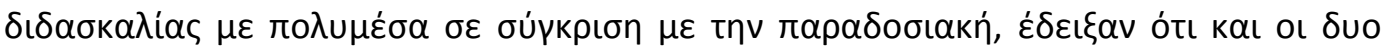

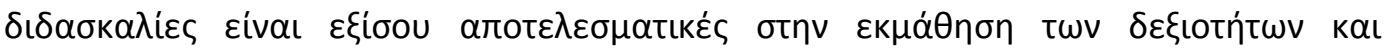

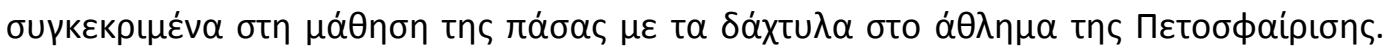

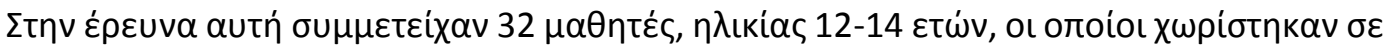

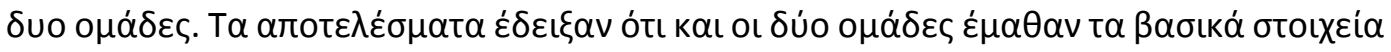

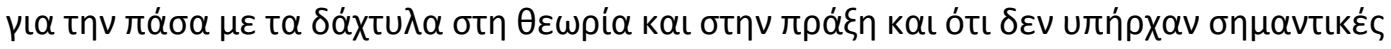

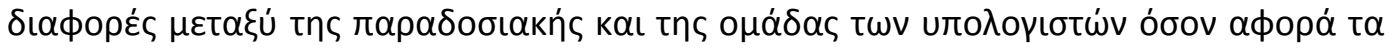

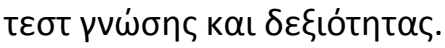




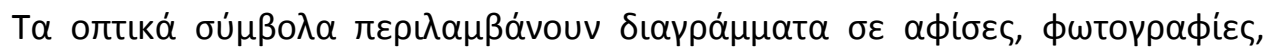

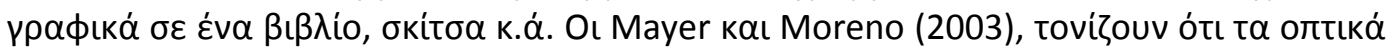

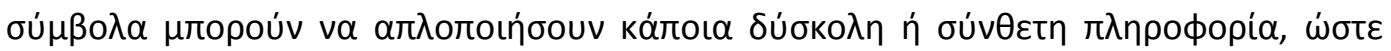

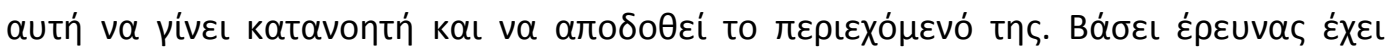

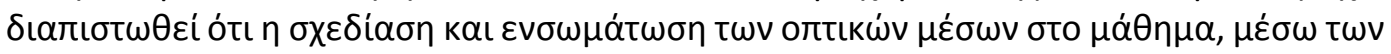

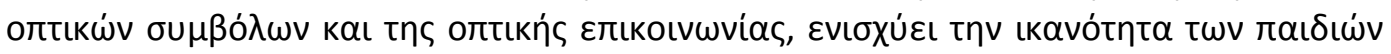

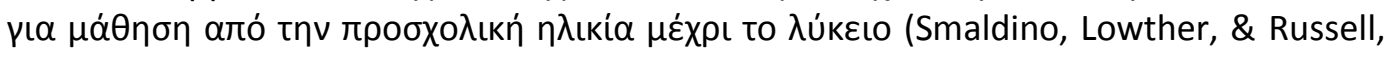
2010).

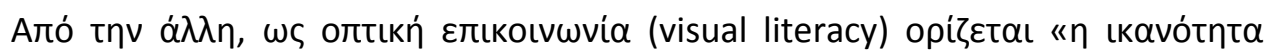

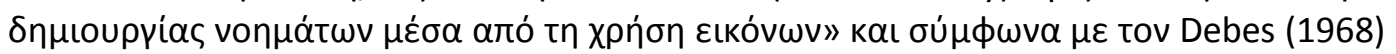

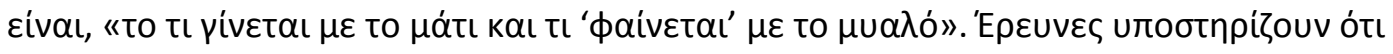

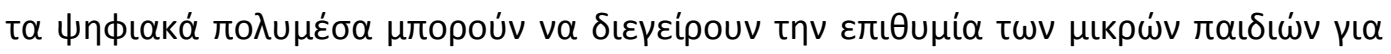

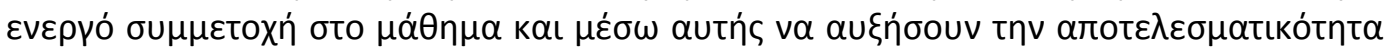

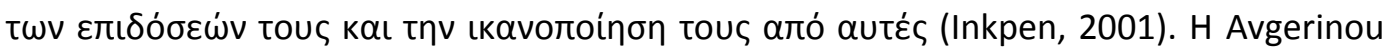

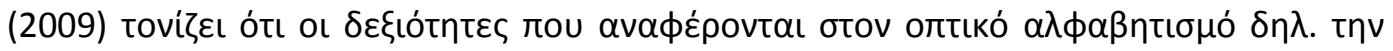

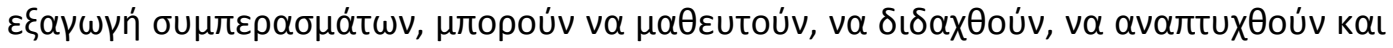

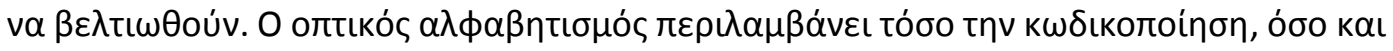

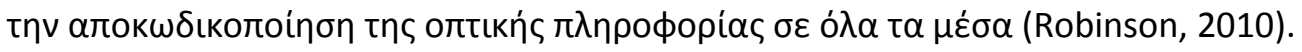

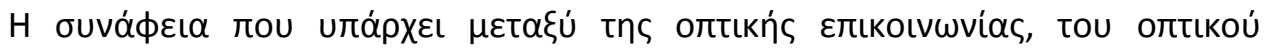

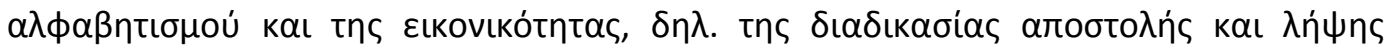

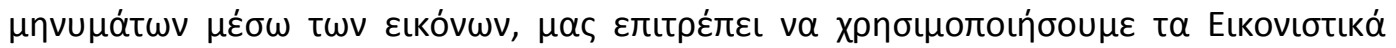

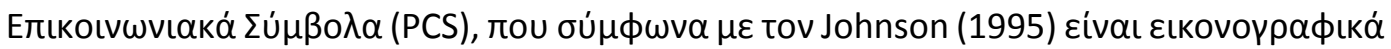

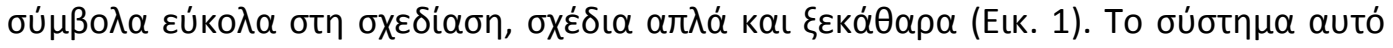

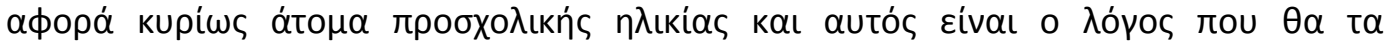

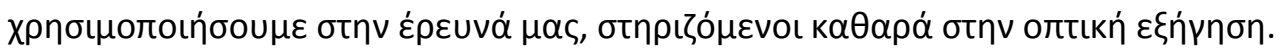

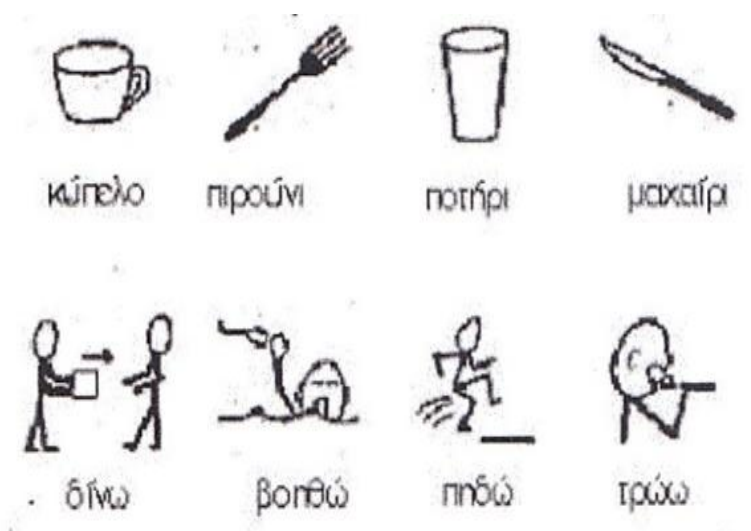

EıKóva 1

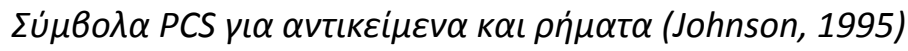

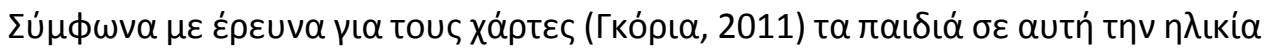

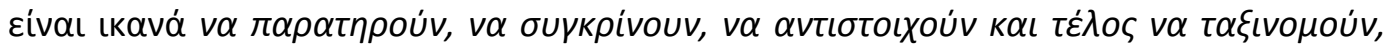

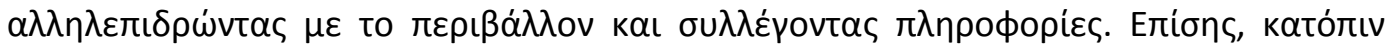




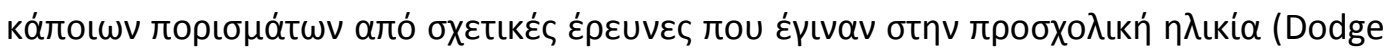

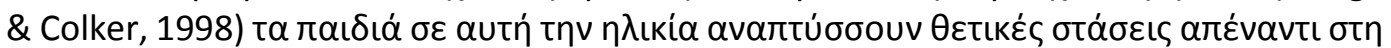

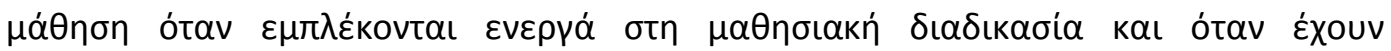

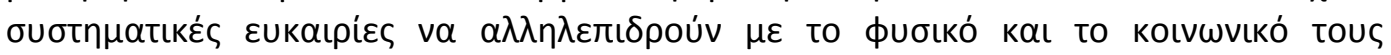
$\pi \varepsilon \rho \iota \beta \dot{\alpha} \lambda \lambda o v$.

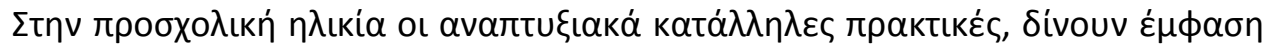

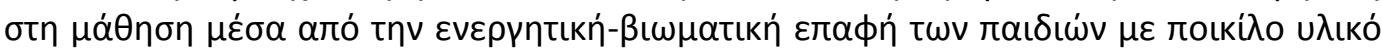

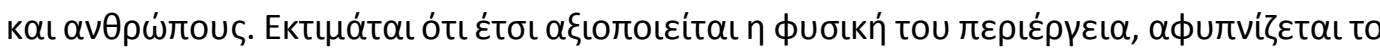

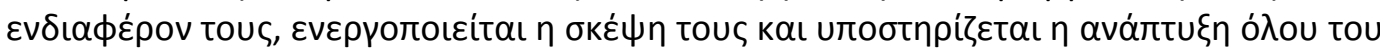

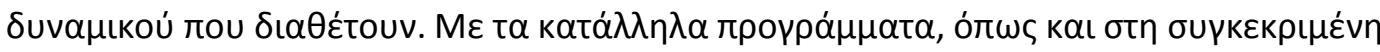

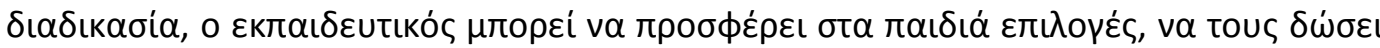

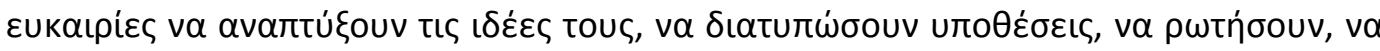

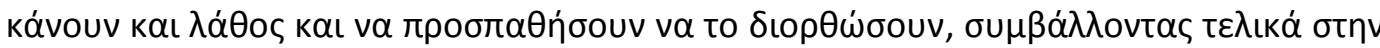

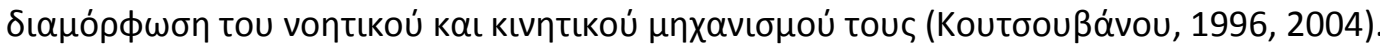

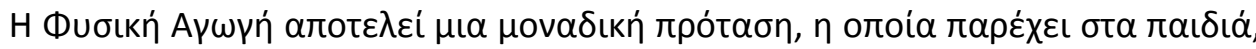

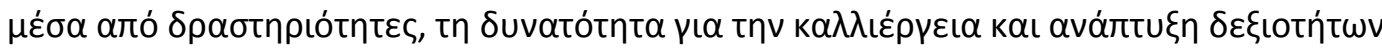

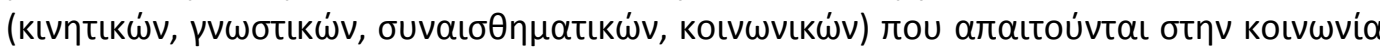

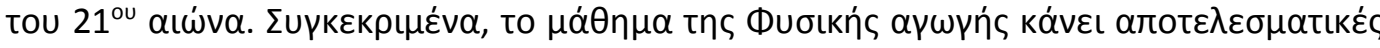

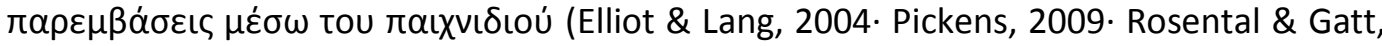

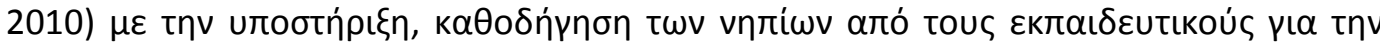

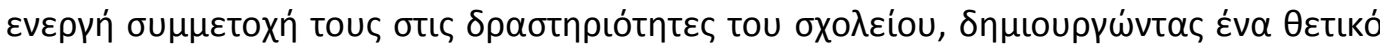

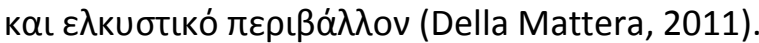

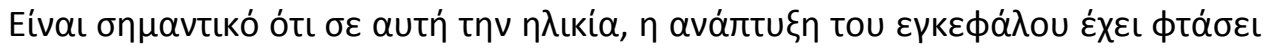

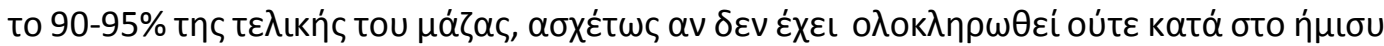

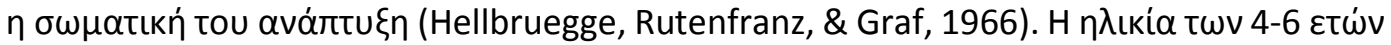

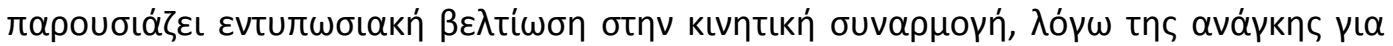

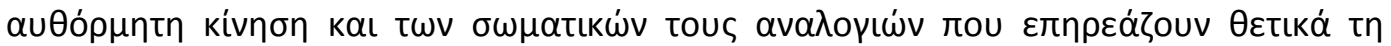

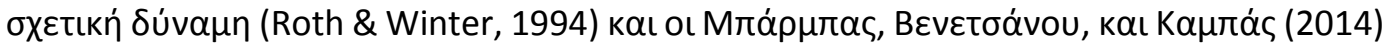

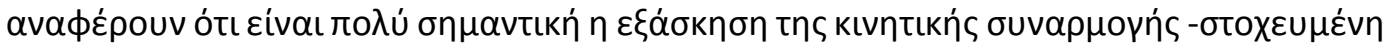

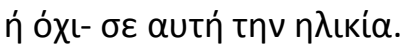

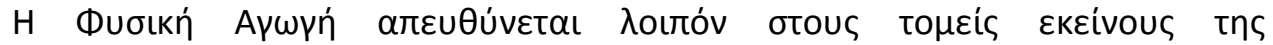

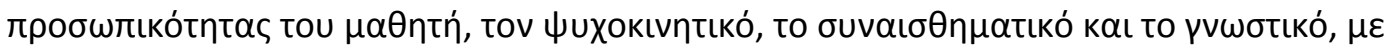

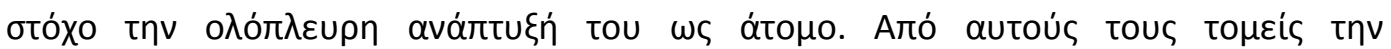

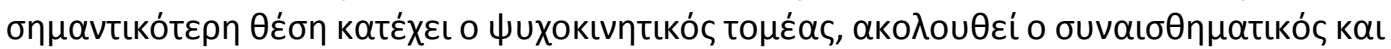

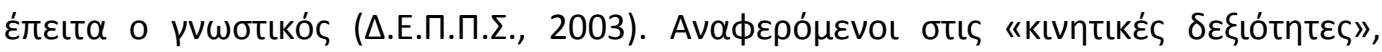

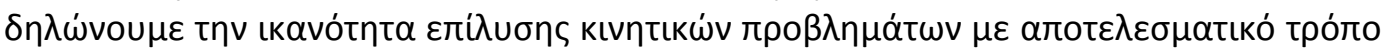

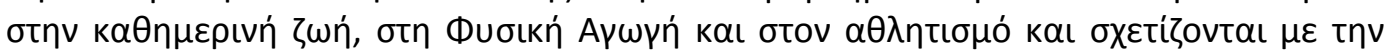

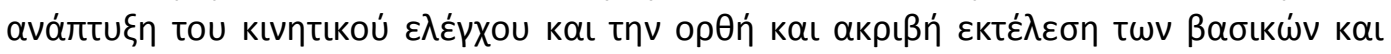

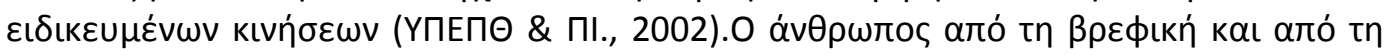

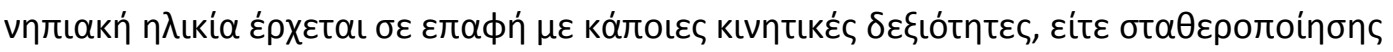

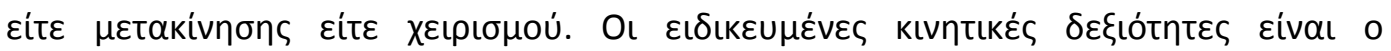

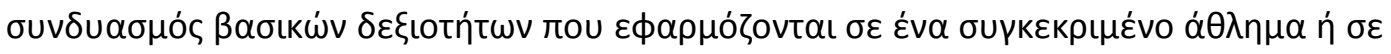

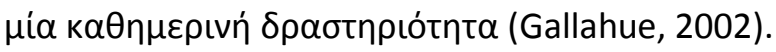

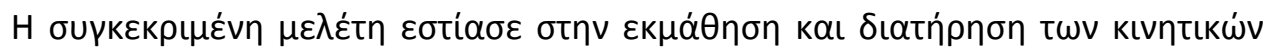

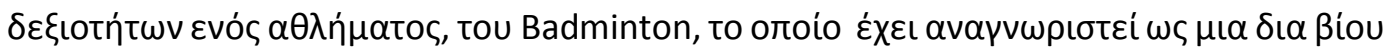




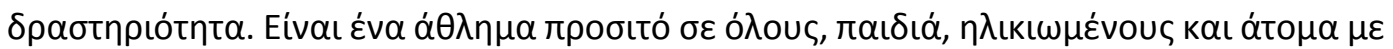

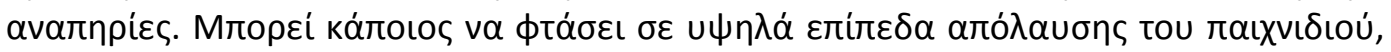

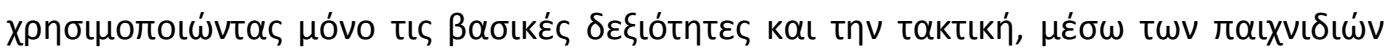
(Wang, 2004).

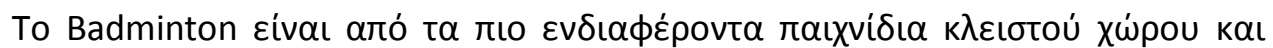

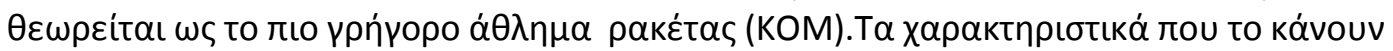

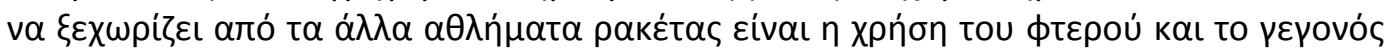

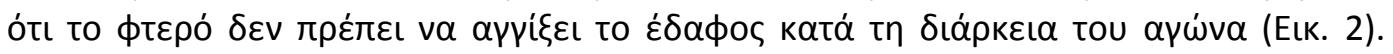

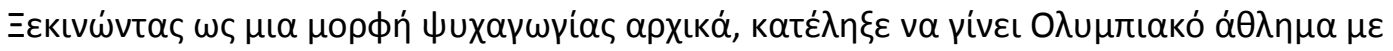

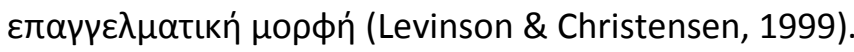
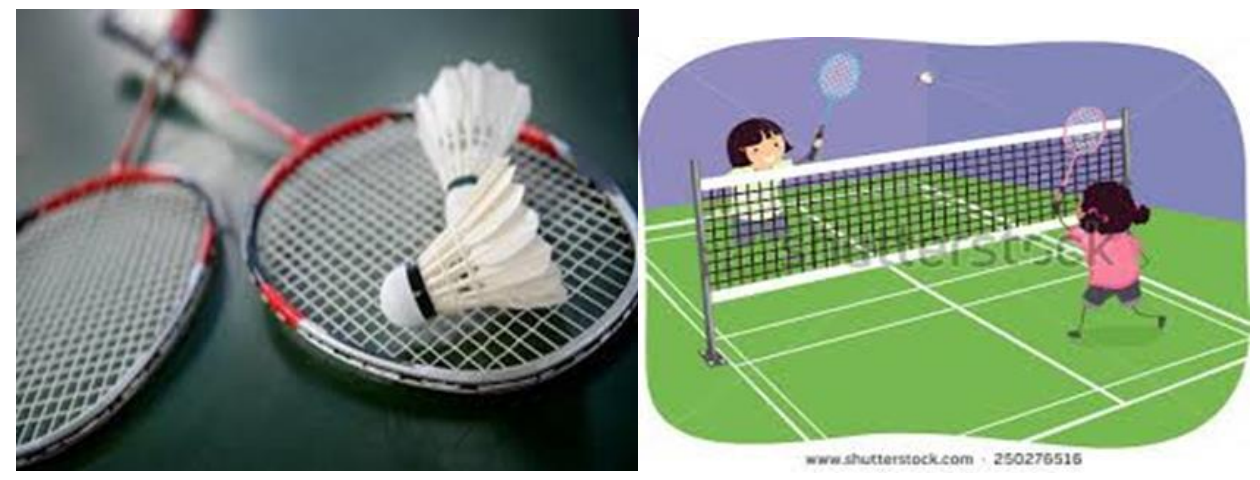

\section{Eıкóva 2}

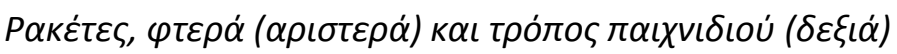

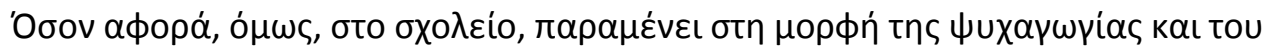

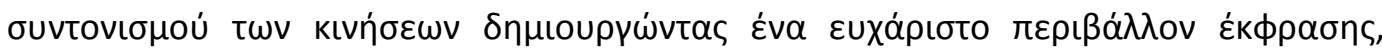

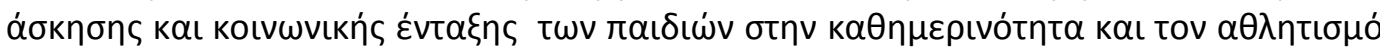

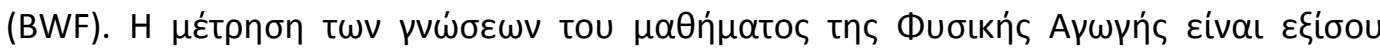

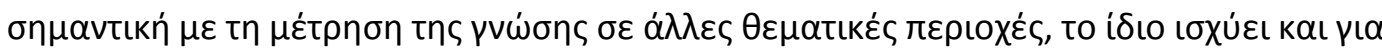

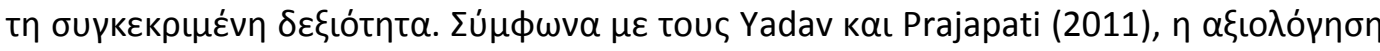

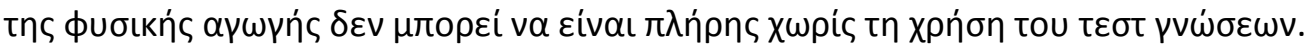

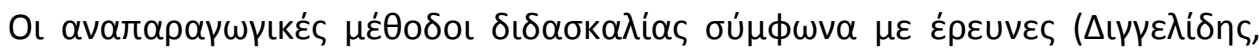

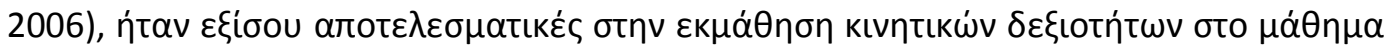

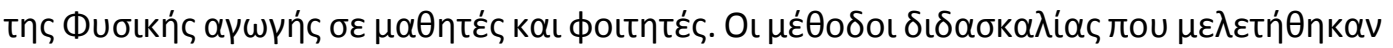

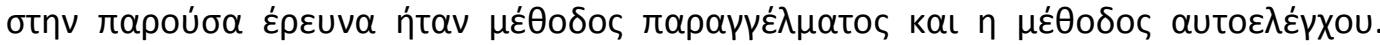

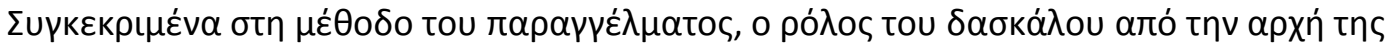

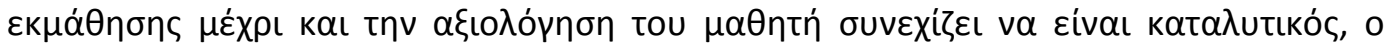

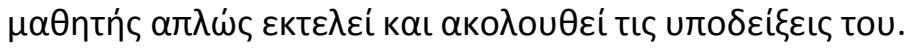

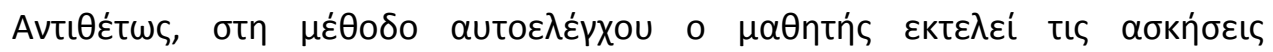

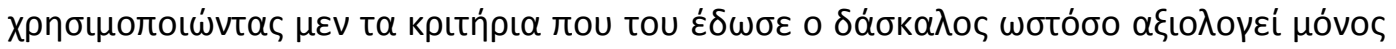

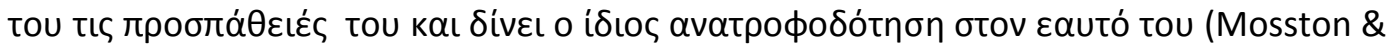

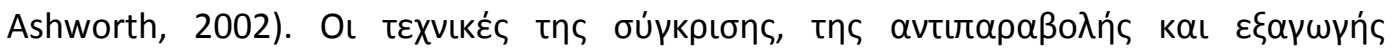

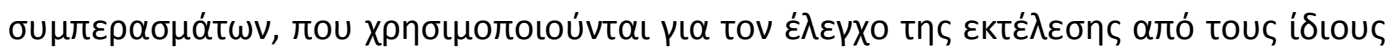




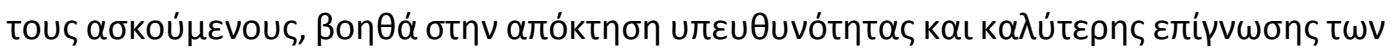

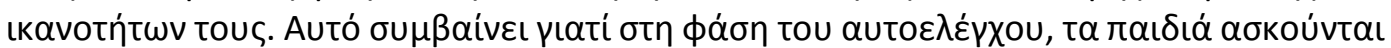

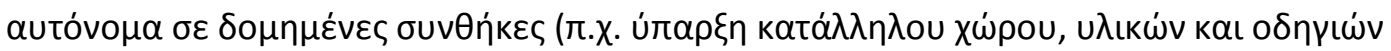

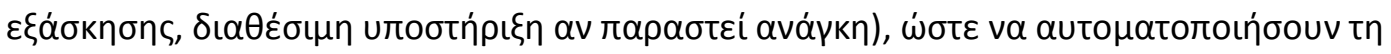
$\delta \varepsilon \xi$ เótnta.

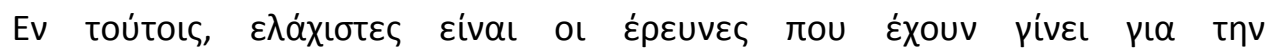

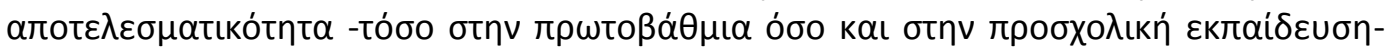

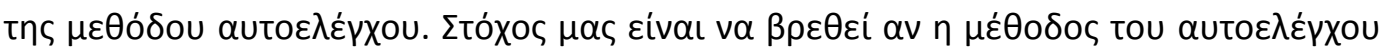

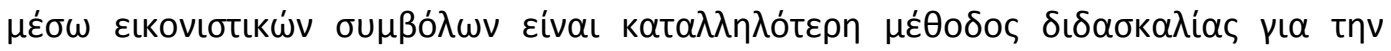

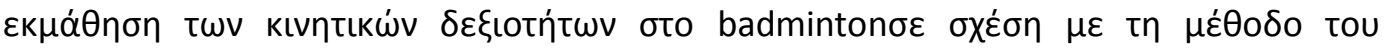

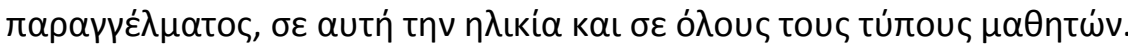

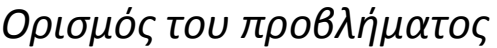

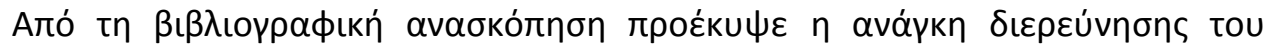

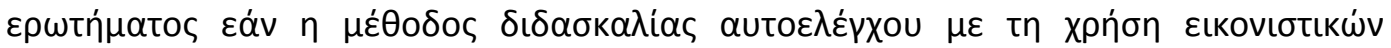

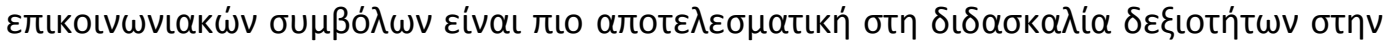

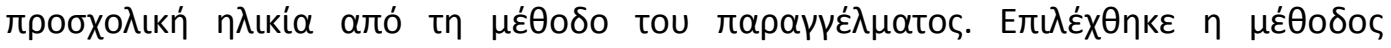

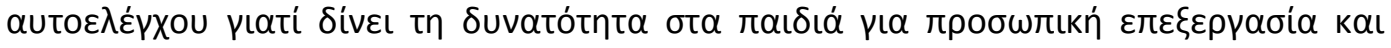

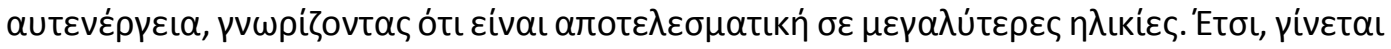

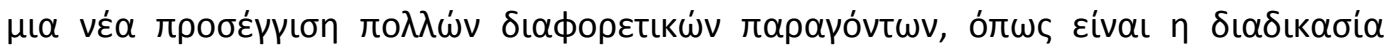

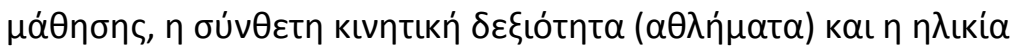

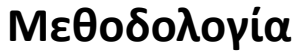

\section{$\Delta \varepsilon i ́ \gamma \mu \alpha$}

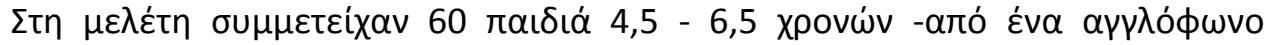

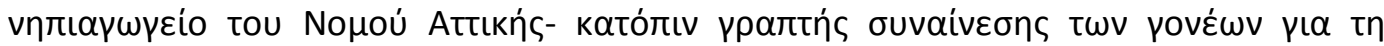

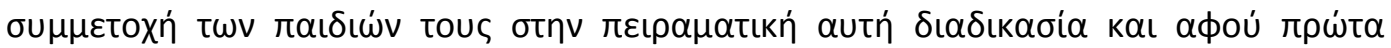

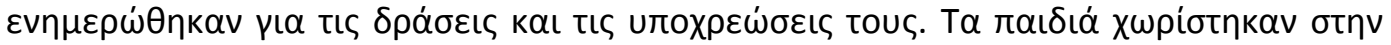

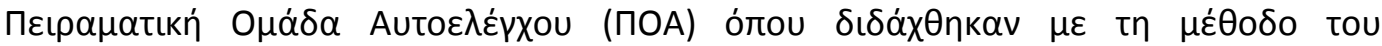

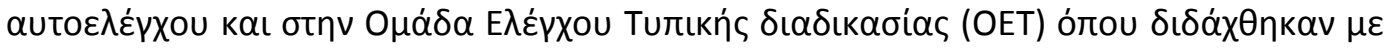

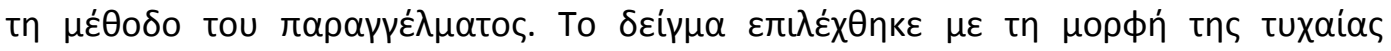

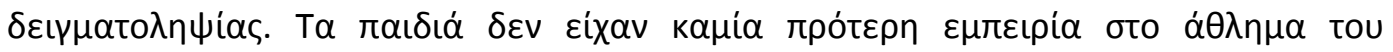
badminton.

\section{$\Delta \iota \alpha \delta \iota \kappa \alpha \sigma i \alpha \pi \alpha \rho \dot{\mu} \mu \beta \alpha \sigma \eta \varsigma$}

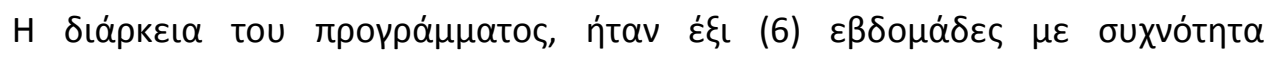

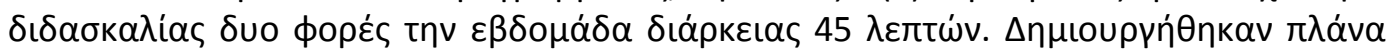

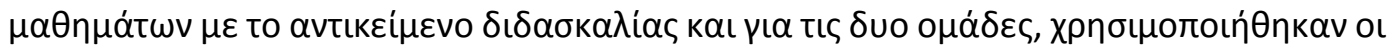

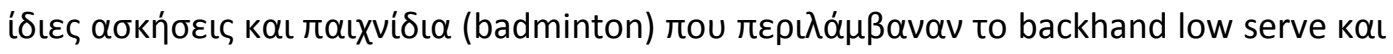

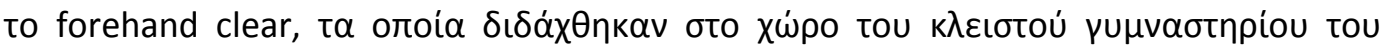

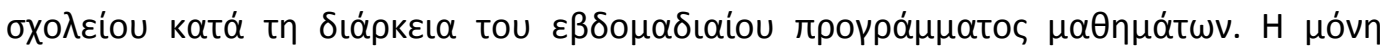

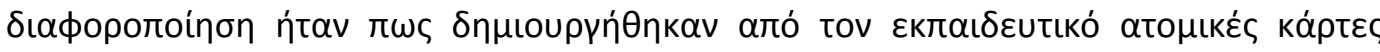

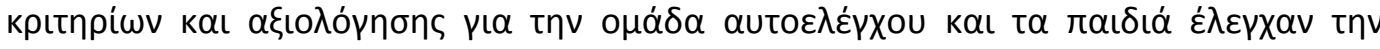

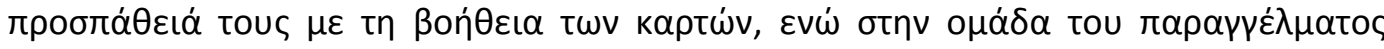

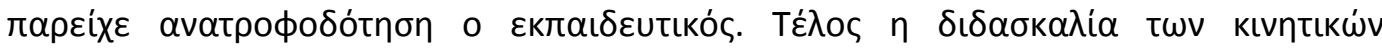

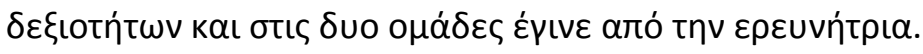




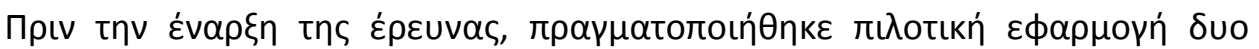

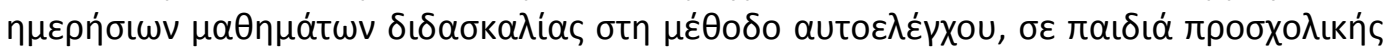

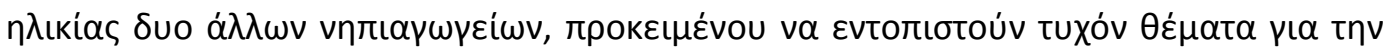

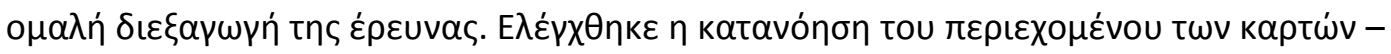

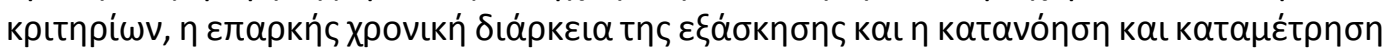

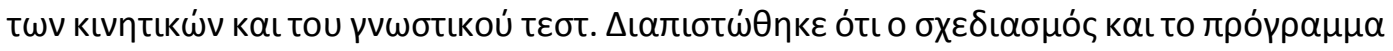

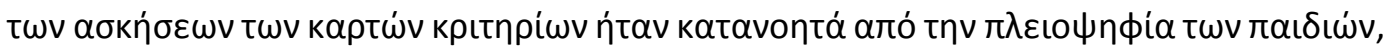

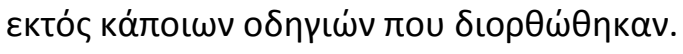

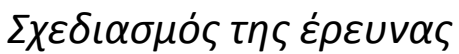

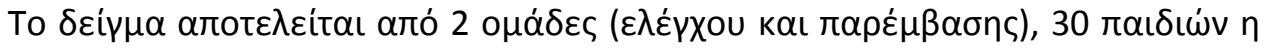

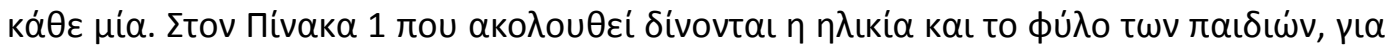

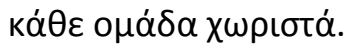

\begin{tabular}{|c|c|c|c|c|c|c|}
\hline & & \multicolumn{2}{|c|}{ OET } & \multicolumn{2}{|c|}{ ПОА } & \multirow[t]{2}{*}{$\mathbf{P}$} \\
\hline & & $\mathbf{N}$ & $\%$ & $\mathbf{N}$ & $\%$ & \\
\hline \multirow{2}{*}{ Фú̉o } & Aүópıа & 16 & 53,3 & 14 & 46,7 & \multirow[t]{2}{*}{$0,606^{*}$} \\
\hline & Корітбıа & 14 & 46,7 & 16 & 53,3 & \\
\hline$H \lambda_{L}$ & 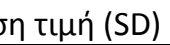 & \multicolumn{2}{|c|}{$5,5(0,8)$} & \multicolumn{2}{|c|}{$5,8(0,5)$} & $0,089 * *$ \\
\hline
\end{tabular}

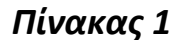

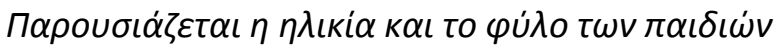

*Pearson'sx²test

**Student's-test

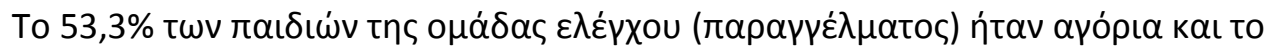

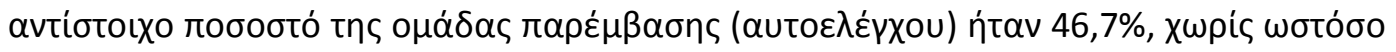

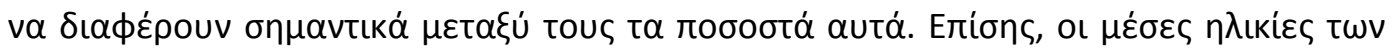

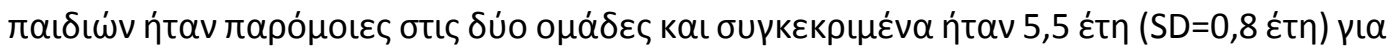

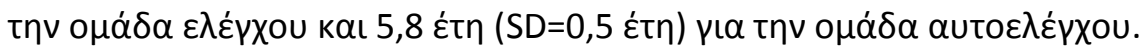

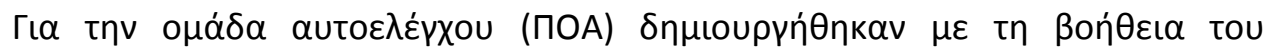

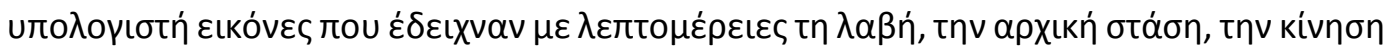

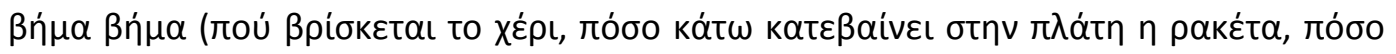

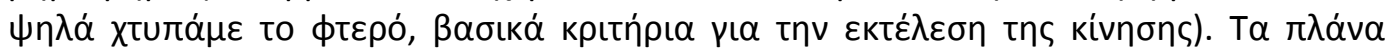

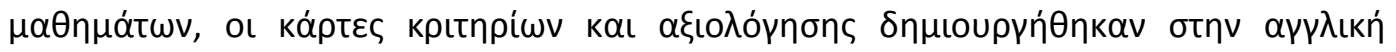

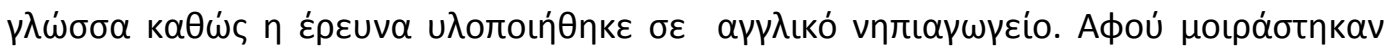

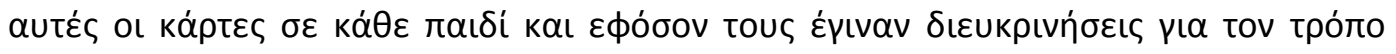

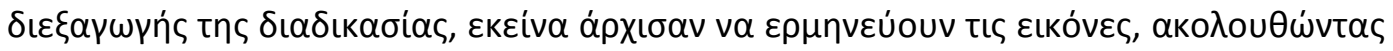

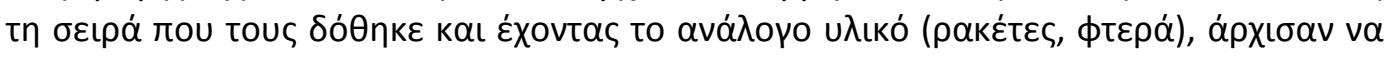

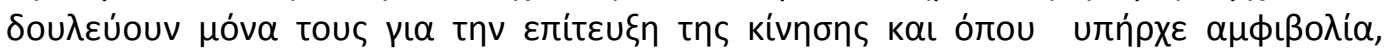

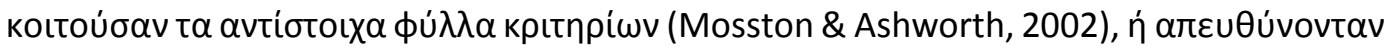

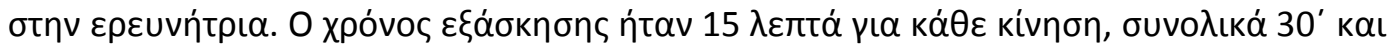

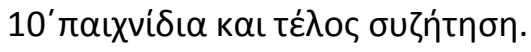




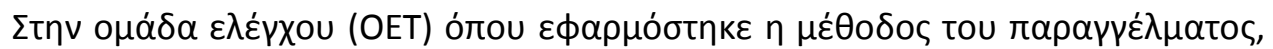

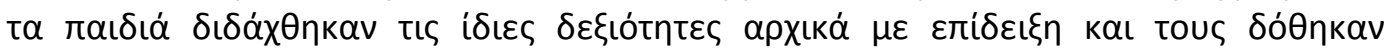

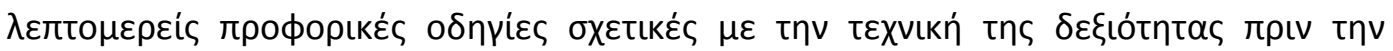

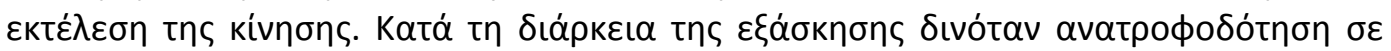

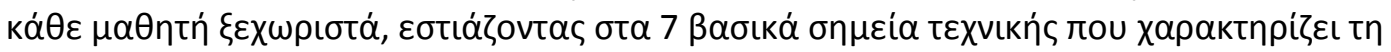

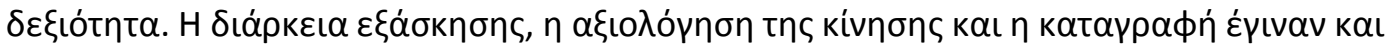

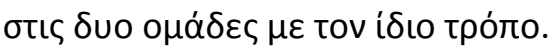

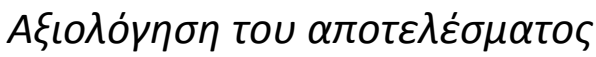

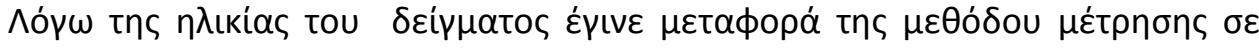

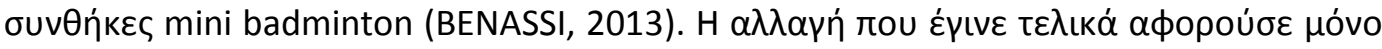

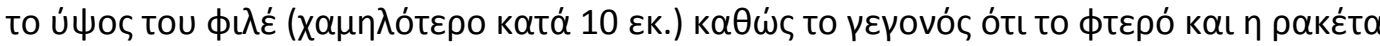

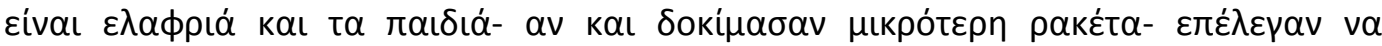

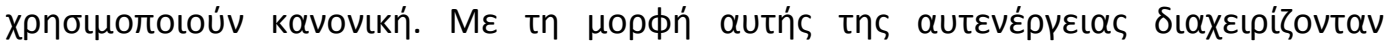

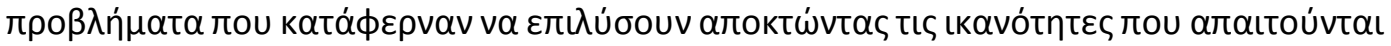

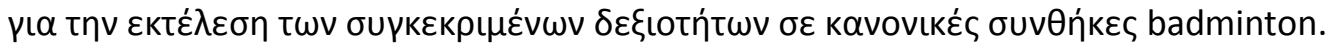

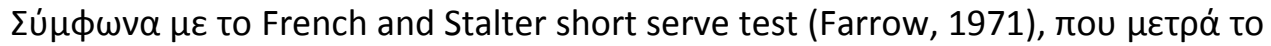

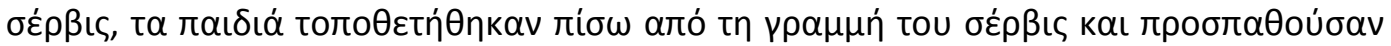

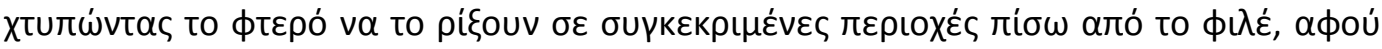

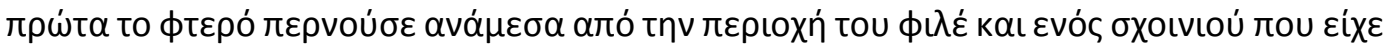

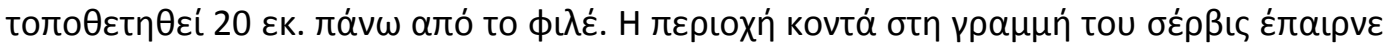

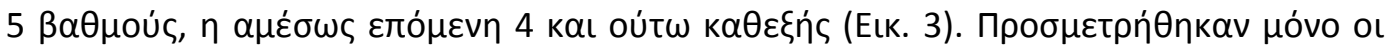

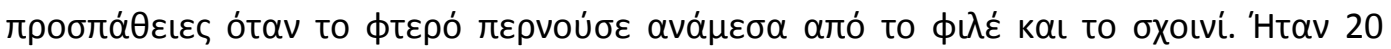

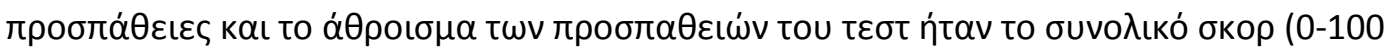

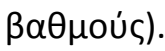

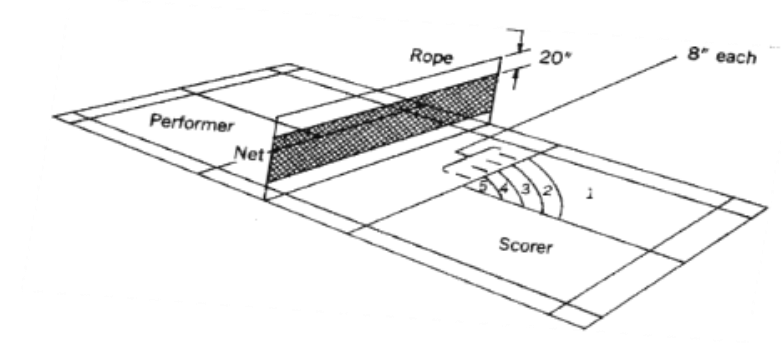

Eıкóva3

$\Sigma \chi \varepsilon \delta\llcorner\dot{\alpha} \gamma \rho \alpha \mu \mu \alpha$ tou French and Stalter short serve test $(1949,1989)$

$\sum u ́ \mu \phi \omega v \alpha \mu \varepsilon$ to French and Stalter forehand clear test (Farrow, 1971), ol

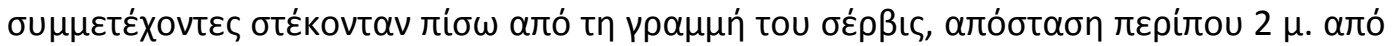

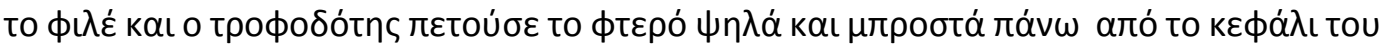

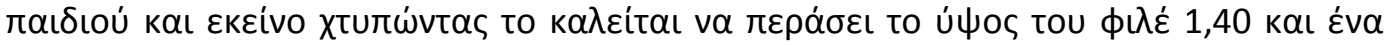

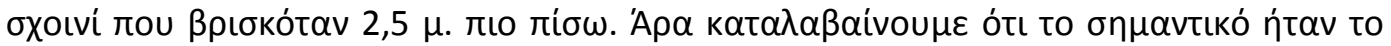

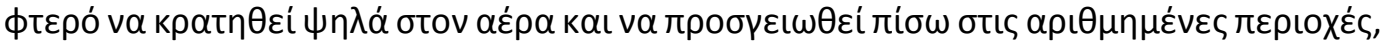

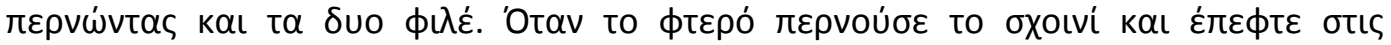

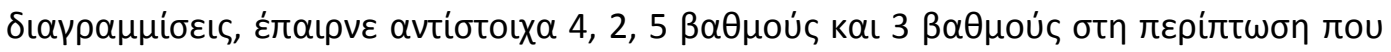

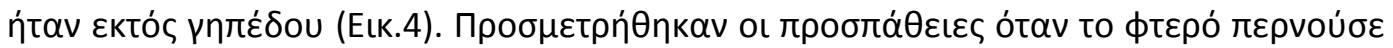




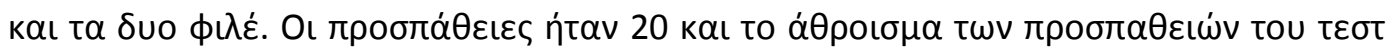

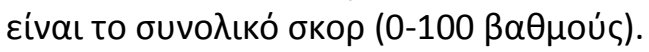

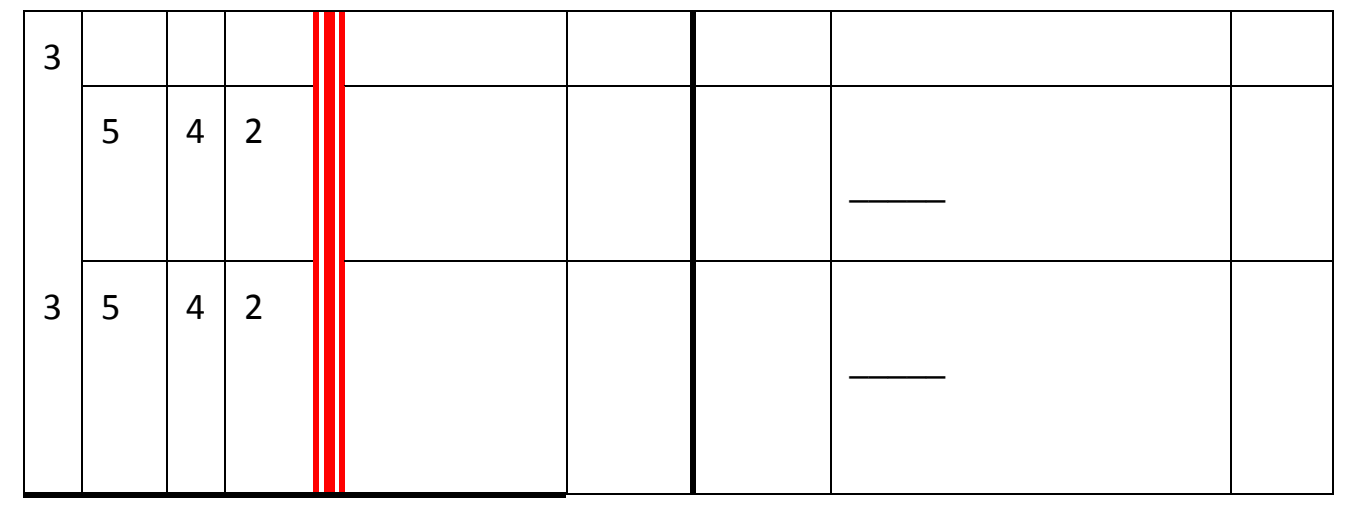

\section{Eıkóva 4}

$\Sigma \chi \varepsilon \delta\llcorner\dot{\alpha} \gamma \rho \alpha \mu \mu \alpha$ tou French and Stalter clear test $(1949,1989)$

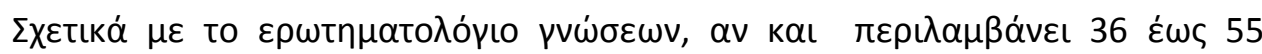

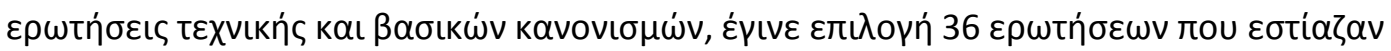

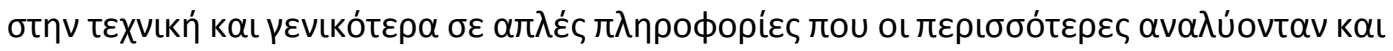

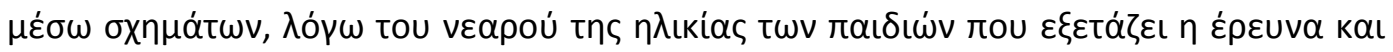

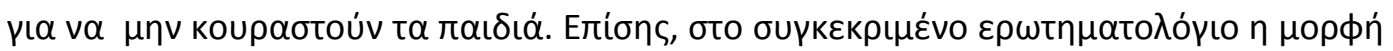

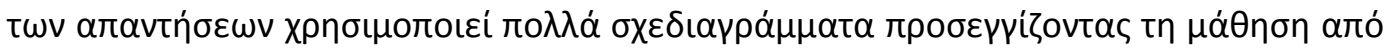

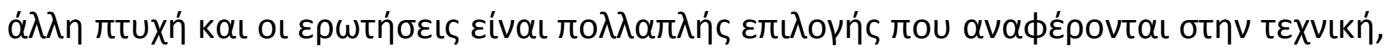

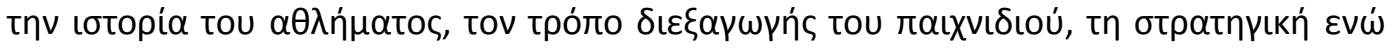

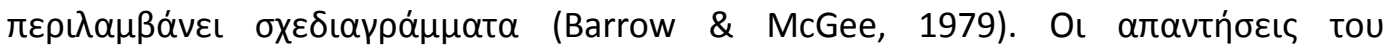

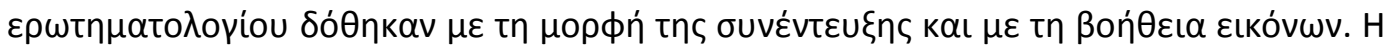

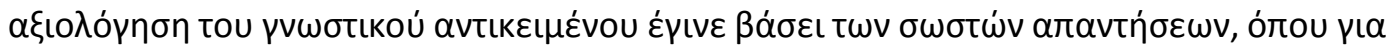

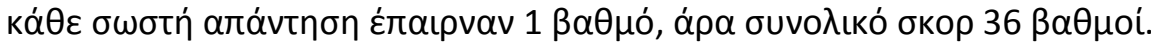

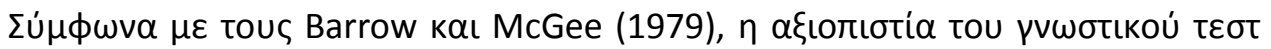

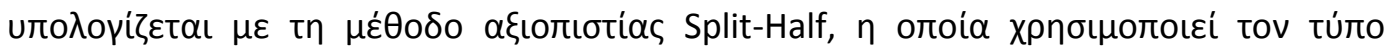

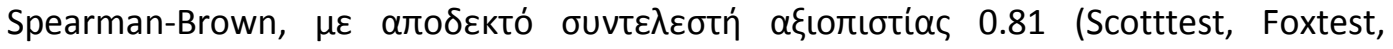

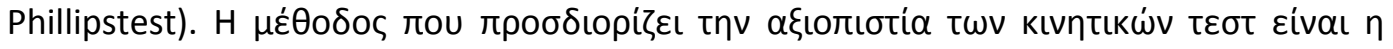

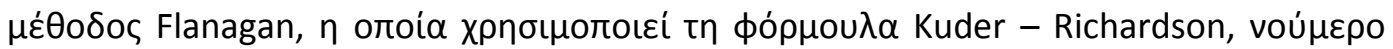

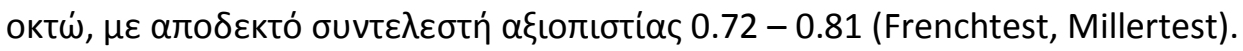

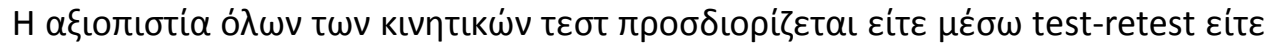

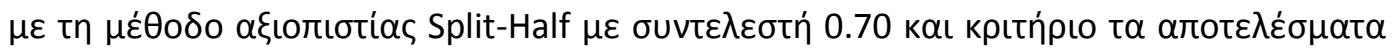

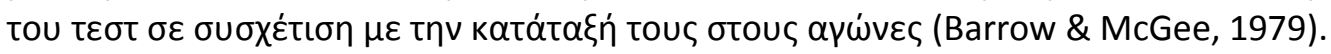

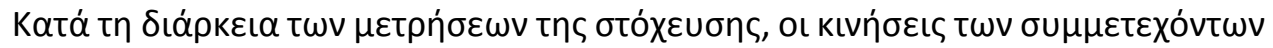

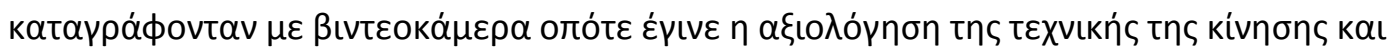

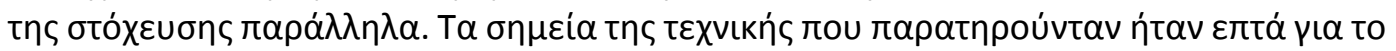

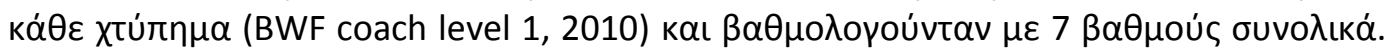

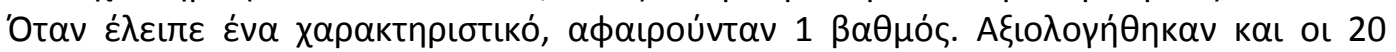




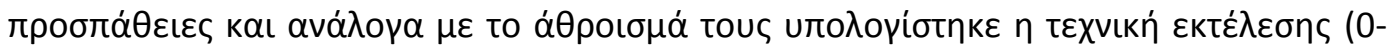
$140 \beta \alpha \theta \mu o i ́)$.

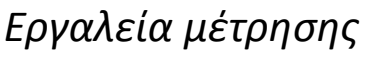

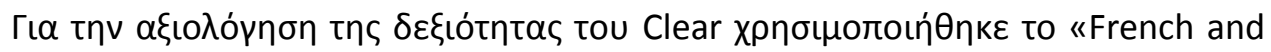

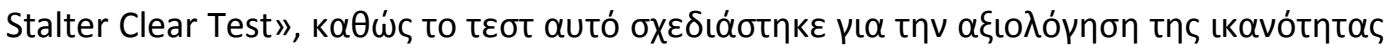

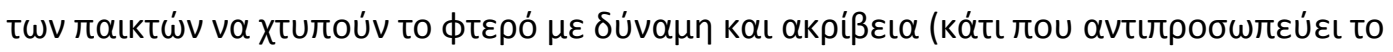

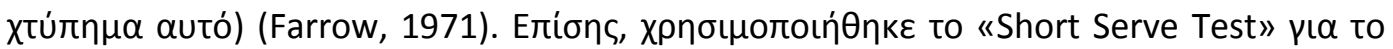

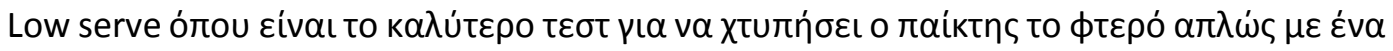

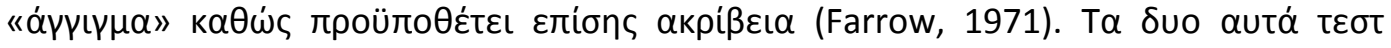

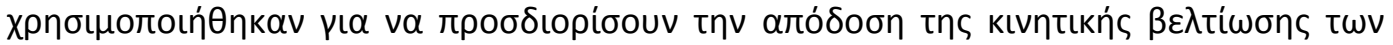

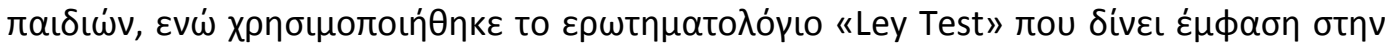

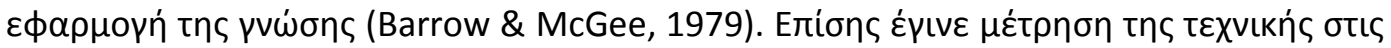

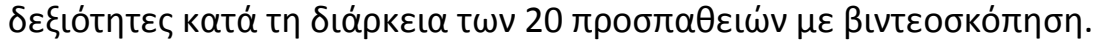

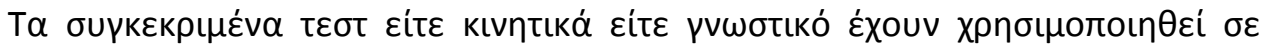

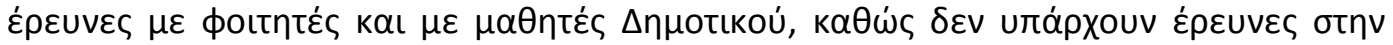

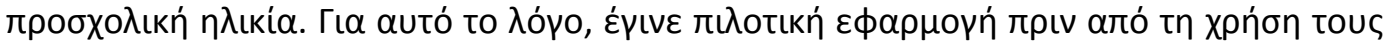

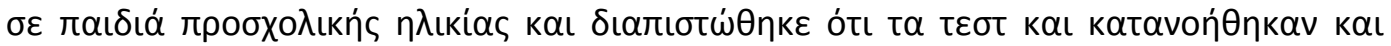

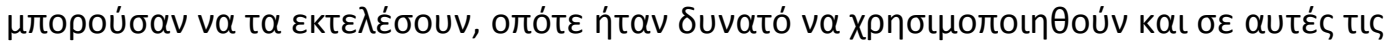
$\eta \lambda \iota \kappa i \varepsilon \varsigma$.

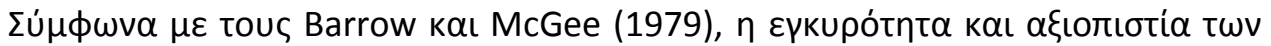

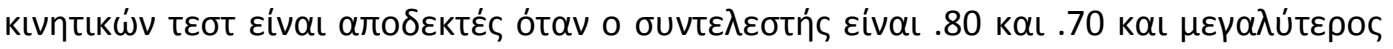

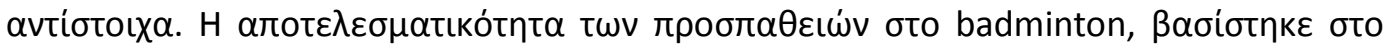

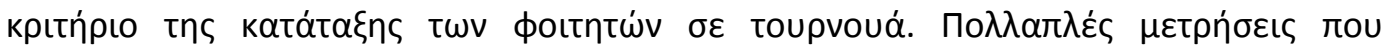

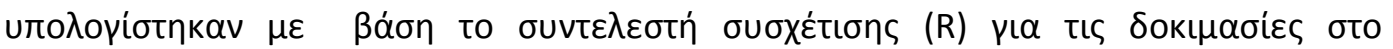

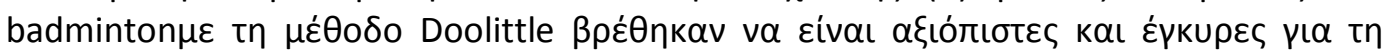

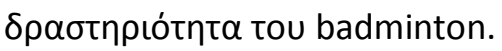

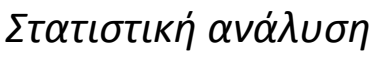

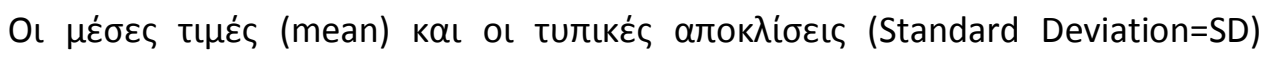

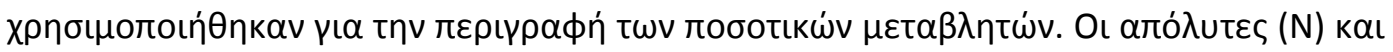

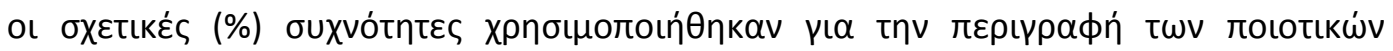

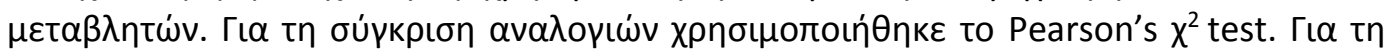

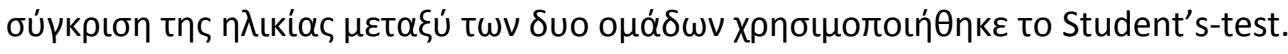

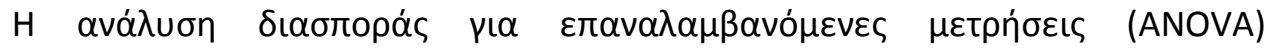

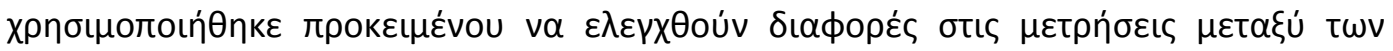

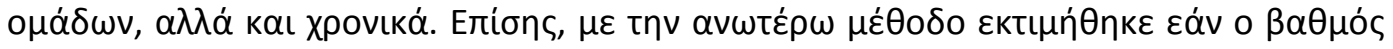

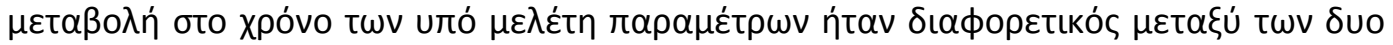

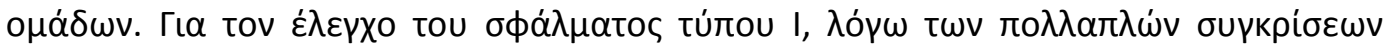

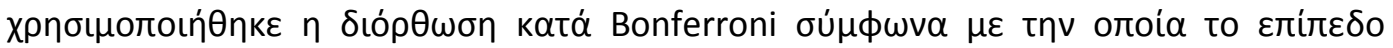

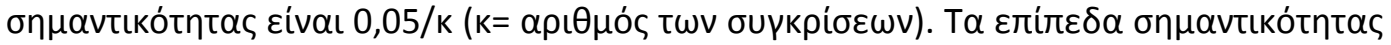

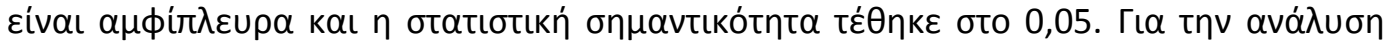

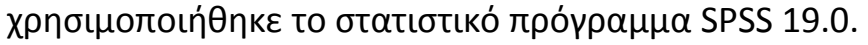




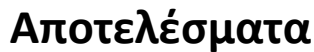

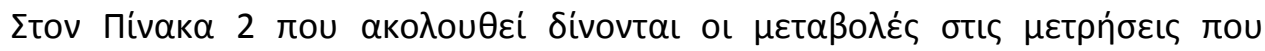

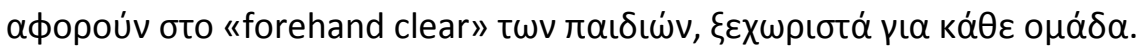

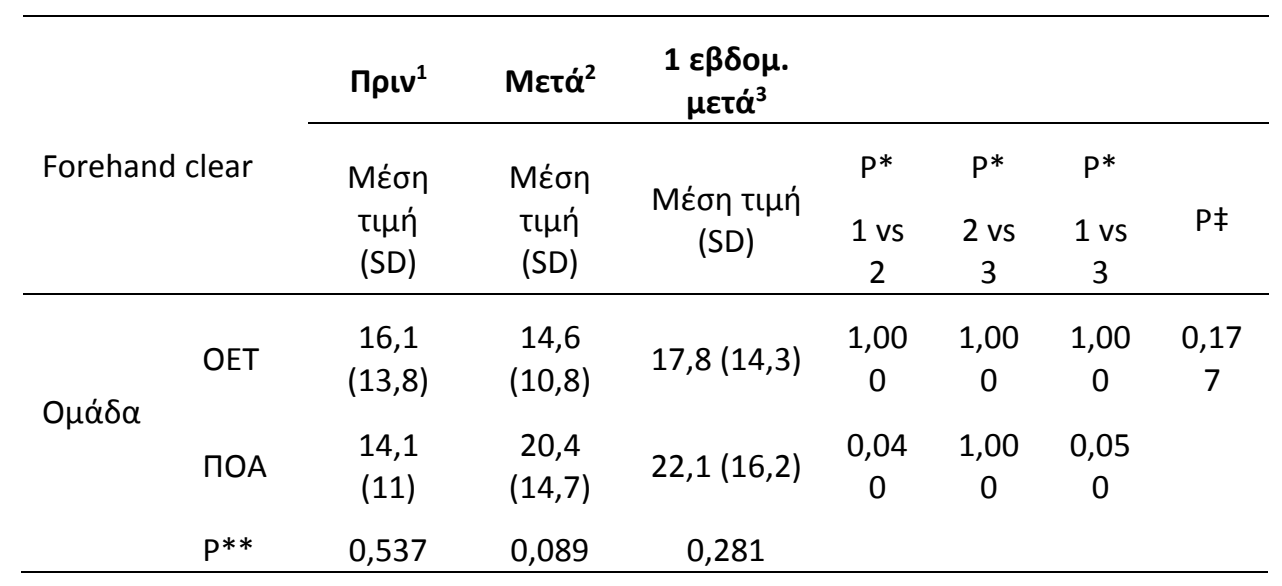

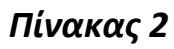

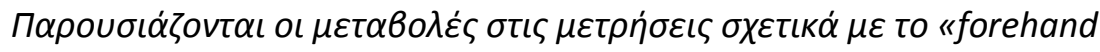

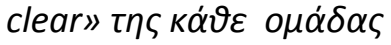

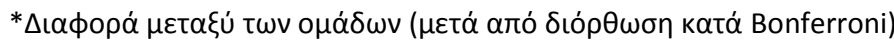

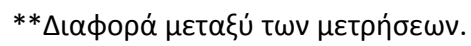

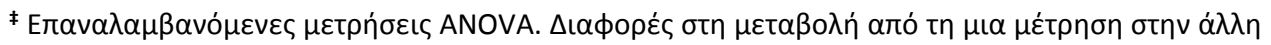

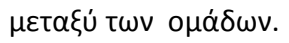

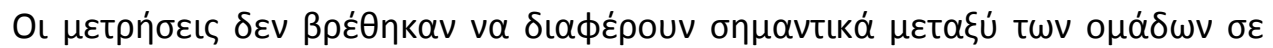

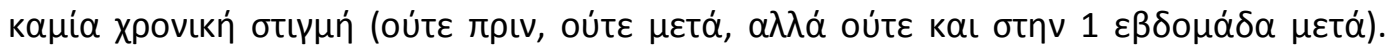

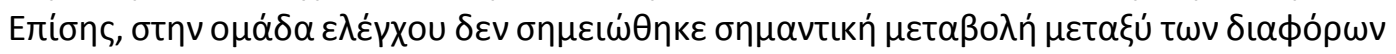

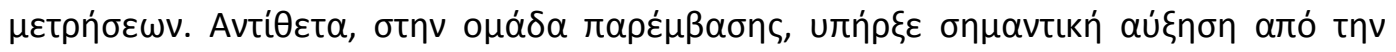

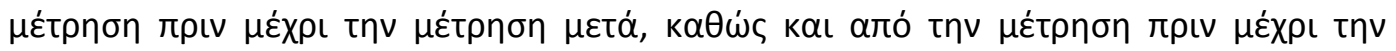

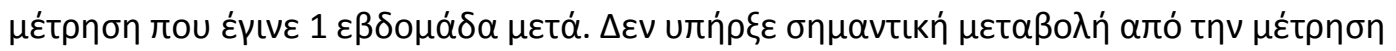

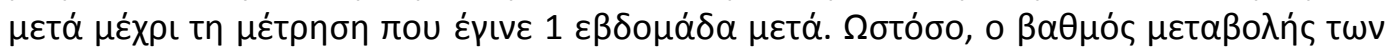

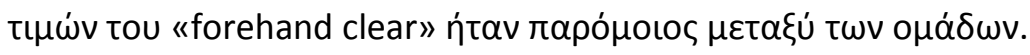

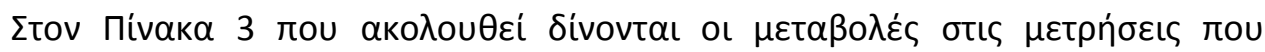

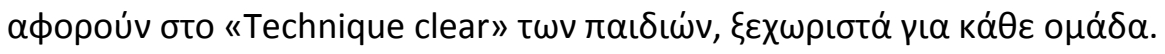




\begin{tabular}{|c|c|c|c|c|c|c|c|c|}
\hline \multirow{2}{*}{\multicolumn{2}{|c|}{$\begin{array}{l}\text { Technique } \\
\text { clear }\end{array}$}} & $\Pi \rho ı v^{1}$ & Metá ${ }^{2}$ & $\begin{array}{c}1 \varepsilon \beta \delta о \mu . \\
\mu \varepsilon \tau \alpha^{3}\end{array}$ & & & & \multirow[b]{2}{*}{$\mathrm{P} \ddagger$} \\
\hline & & 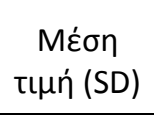 & 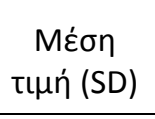 & 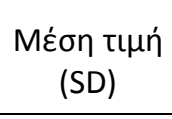 & $\begin{array}{c}\mathrm{P}^{*} \\
1 \mathrm{vs2}\end{array}$ & $\begin{array}{c}P^{*} \\
2 \text { vs } 3 \\
\end{array}$ & $\begin{array}{c}\mathrm{P}^{*} \\
1 \mathrm{vs3}\end{array}$ & \\
\hline \multirow{3}{*}{ O$\mu \alpha \dot{\alpha} \delta \alpha$} & OET & $\begin{array}{c}67,7 \\
(19,5)\end{array}$ & $\begin{array}{c}83,3 \\
(14,9)\end{array}$ & $80,3(19,5)$ & $<0,001$ & 0,958 & $<0,001$ & \multirow[t]{3}{*}{0,130} \\
\hline & ПОА & $\begin{array}{c}73,4 \\
(21,5)\end{array}$ & $\begin{array}{c}87,7 \\
(25,3)\end{array}$ & $92,7(20,7)$ & $<0,001$ & 0,288 & $<0,001$ & \\
\hline & $\mathrm{P} * *$ & 0,284 & 0,419 & 0,021 & & & & \\
\hline
\end{tabular}

Піvaкаৎ 3

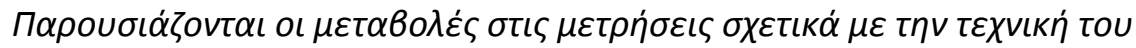

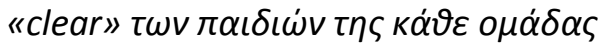

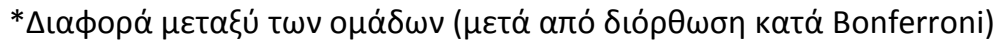

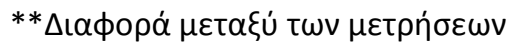

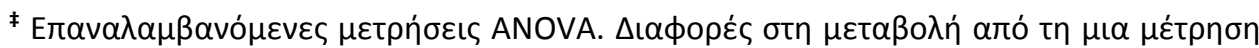

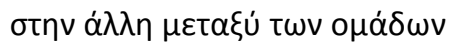

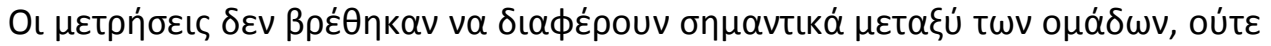

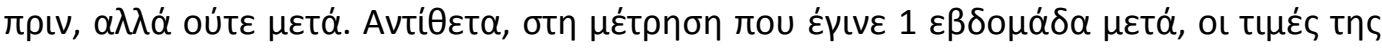

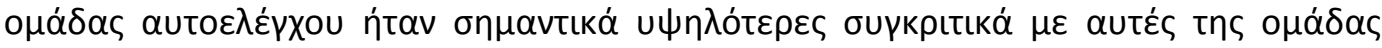

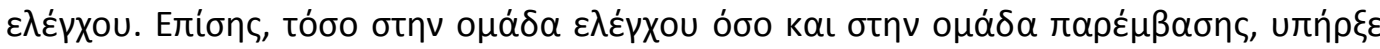

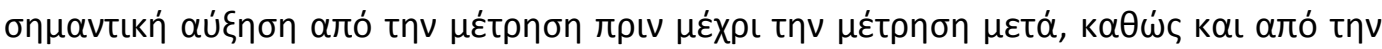

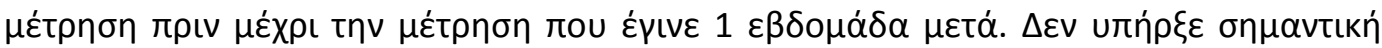

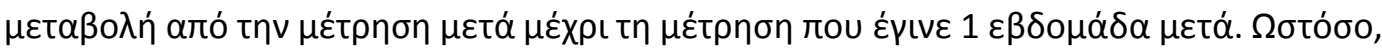

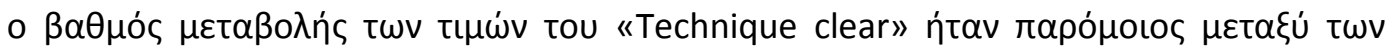

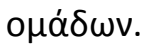

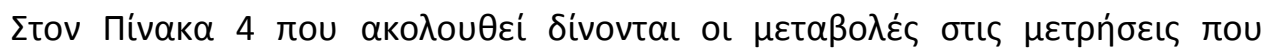

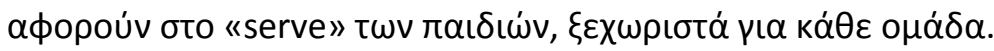




\begin{tabular}{|c|c|c|c|c|c|c|c|c|}
\hline \multirow[b]{2}{*}{ Serve } & & $\Pi \rho v^{1}$ & Metád & $\begin{array}{c}1 \varepsilon \beta \delta o \mu . \\
\mu \varepsilon \tau \dot{\alpha}^{3}\end{array}$ & & & & \\
\hline & & $\begin{array}{c}\text { Mźon } \\
\tau \iota \mu \hat{~} \\
\text { (SD) }\end{array}$ & $\begin{array}{l}\text { Mźon } \\
\tau\langle\mu \eta ́ \\
\text { (SD) }\end{array}$ & $\begin{array}{c}\text { Mźon } \\
\tau \iota \mu \eta ́(S D)\end{array}$ & $\begin{array}{c}\mathrm{P}^{*} \\
1 \text { vs } \\
2\end{array}$ & $\begin{array}{c}\mathrm{P}^{*} \\
2 \text { vs } \\
3\end{array}$ & $\begin{array}{c}\mathrm{P}^{*} \\
1 \text { vs } \\
3\end{array}$ & $P \ddagger$ \\
\hline \multirow[t]{3}{*}{ 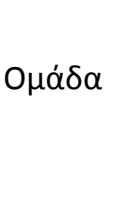 } & OET & $\begin{array}{c}11 \\
(14,8)\end{array}$ & $9,3(6,3)$ & $7,6(6,1)$ & 1,000 & 0,828 & 0,537 & 0,783 \\
\hline & ПОА & $8,5(7,6)$ & $9,2(6,1)$ & $10,9(9,6)$ & 1,000 & 0,800 & 1,000 & \\
\hline & $\mathrm{P}^{* *}$ & 0,414 & 0,983 & 0,122 & & & & \\
\hline
\end{tabular}

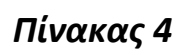

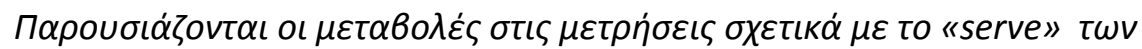

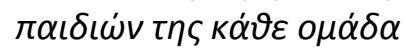

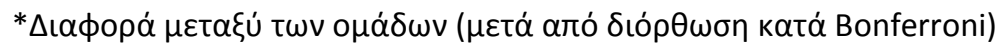

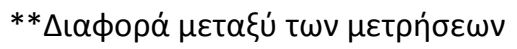

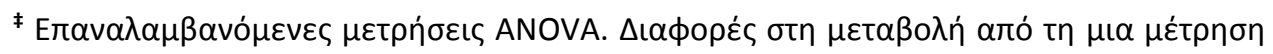

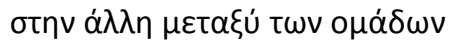

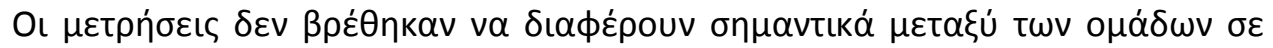

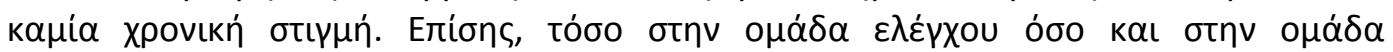
$\pi \alpha \rho \varepsilon \dot{\mu} \mu \alpha \sigma \eta \varsigma, \delta \varepsilon v$ u

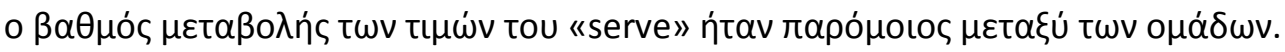

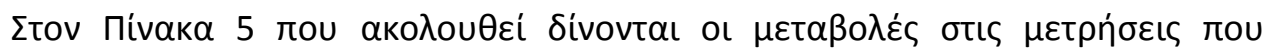

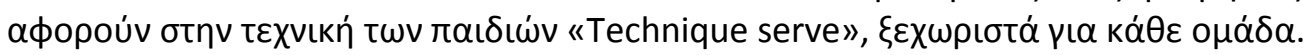

\begin{tabular}{|c|c|c|c|c|c|c|c|c|}
\hline \multirow{2}{*}{$\begin{array}{l}\text { Technique } \\
\text { serve }\end{array}$} & & $\Pi \rho v^{1}$ & 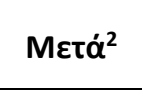 & $\begin{array}{c}1 \varepsilon \beta \delta о \mu . \\
\mu \varepsilon \tau \dot{\alpha}^{3}\end{array}$ & & & & \multirow[b]{2}{*}{$\mathbf{P} \neq$} \\
\hline & & $\begin{array}{l}\text { Mźon } \\
\tau \iota \mu n ́ \\
\text { (SD) }\end{array}$ & $\begin{array}{l}\text { Mźon } \\
\tau i \mu n ́ \\
\text { (SD) }\end{array}$ & 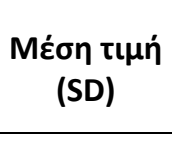 & $\begin{array}{c}\mathrm{P}^{*} \\
1 \text { vs } 2\end{array}$ & $\begin{array}{c}\text { P* } \\
2 \text { vs } 3\end{array}$ & $\begin{array}{c}\text { P* } \\
1 \text { vs } 3\end{array}$ & \\
\hline \multirow{3}{*}{ 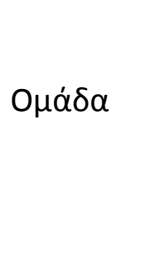 } & OET & $\begin{array}{c}52,7 \\
(15,1)\end{array}$ & $77,2(12)$ & $83,7(15,2)$ & $<0,001$ & 0,157 & $<0,001$ & 0,816 \\
\hline & ПОА & $\begin{array}{c}61,8 \\
(23,7)\end{array}$ & $\begin{array}{c}82,2 \\
(20,3)\end{array}$ & $91,5(20,1)$ & $<0,001$ & 0,019 & $<0,001$ & \\
\hline & $\mathrm{P}^{* *}$ & 0,081 & 0,250 & 0,095 & & & & \\
\hline
\end{tabular}

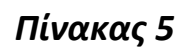

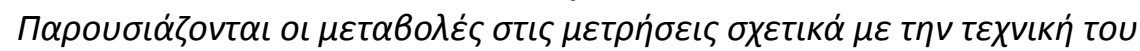

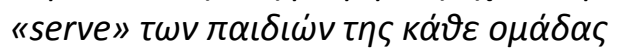

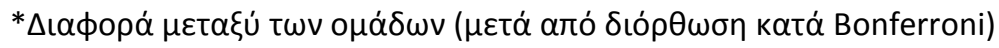




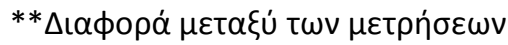

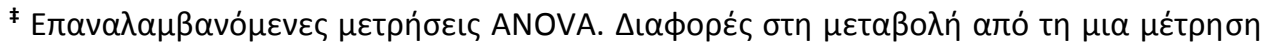

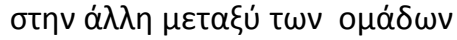

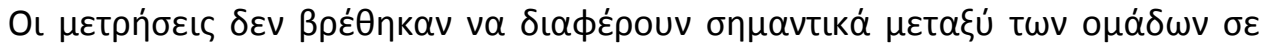

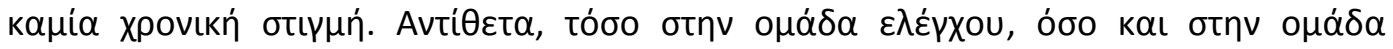

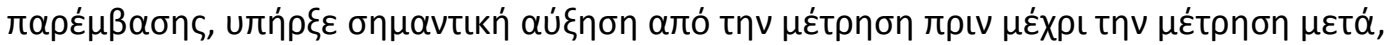

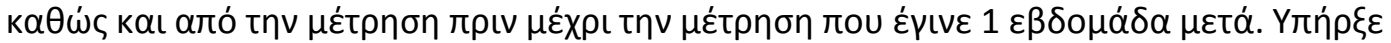

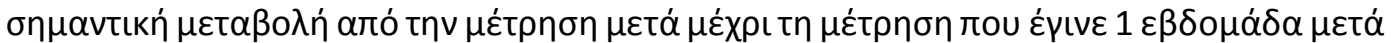

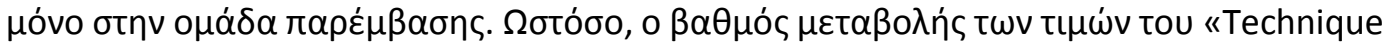

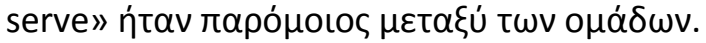

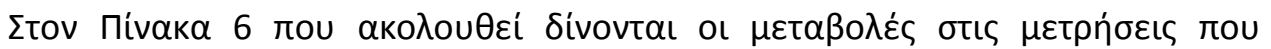

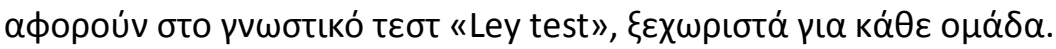

\begin{tabular}{|c|c|c|c|c|c|c|c|c|}
\hline \multirow{2}{*}{ Ley test } & & $\Pi \rho v^{1}$ & Metód & $\begin{array}{c}1 \varepsilon \beta \delta o \mu . \\
\mu \varepsilon \tau \alpha^{3}\end{array}$ & & & & \\
\hline & & 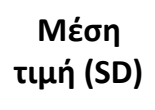 & 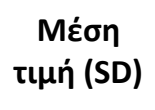 & 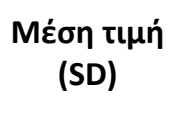 & $\begin{array}{c}\text { P* } \\
1 \text { vs } 2\end{array}$ & $\begin{array}{c}\mathrm{P}^{*} \\
2 \text { vs } 3\end{array}$ & $\begin{array}{c}P^{*} \\
1 \text { vs } 3\end{array}$ & $\mathbf{P} \neq$ \\
\hline \multirow{3}{*}{ 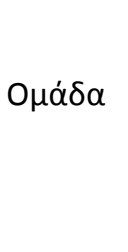 } & OET & $8,4(2,9)$ & $19,4(3,3)$ & $22,5(2,2)$ & $\begin{array}{c}<0,00 \\
1\end{array}$ & $\begin{array}{c}<0,00 \\
1\end{array}$ & $\begin{array}{c}<0,00 \\
1\end{array}$ & $\begin{array}{c}0,00 \\
3\end{array}$ \\
\hline & $\begin{array}{l}\text { חO } \\
\mathrm{A}\end{array}$ & $9(3,3)$ & $16,9(3,3)$ & $22,3(3,2)$ & $\begin{array}{c}<0,00 \\
1\end{array}$ & $\begin{array}{c}<0,00 \\
1\end{array}$ & $\begin{array}{c}<0,00 \\
1\end{array}$ & \\
\hline & $\mathrm{P}^{* *}$ & 0,483 & 0,005 & 0,777 & & & & \\
\hline
\end{tabular}

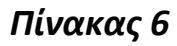

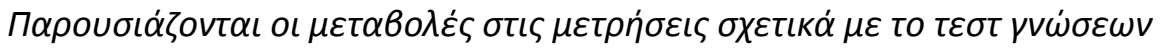

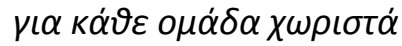

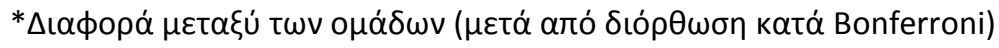

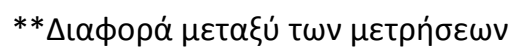

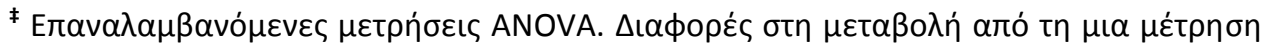

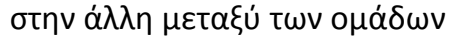

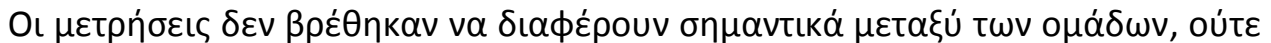

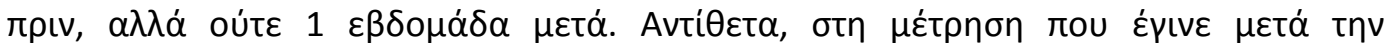

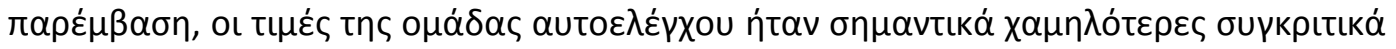

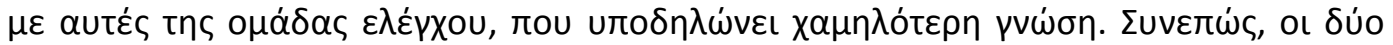

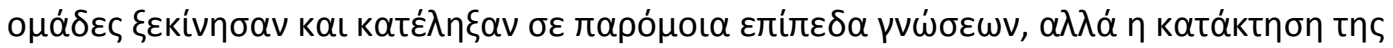

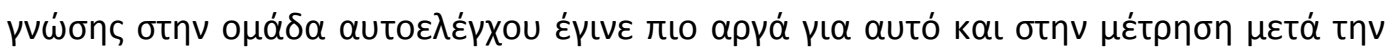

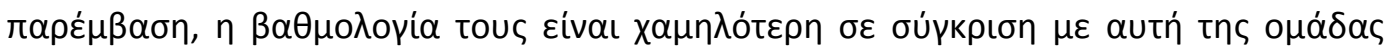
$\varepsilon \lambda \varepsilon \dot{\gamma X O u}$. 


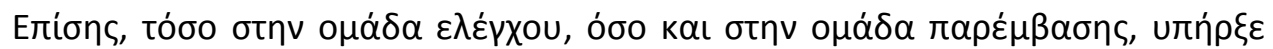

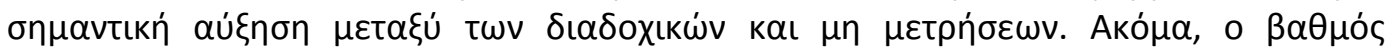

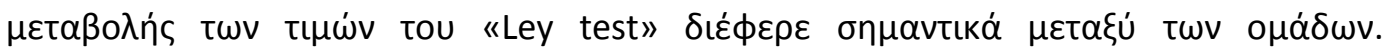

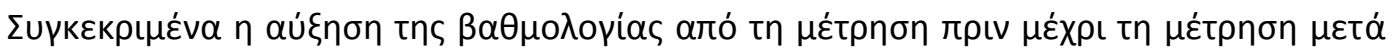

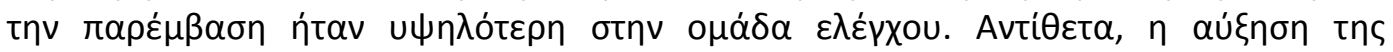

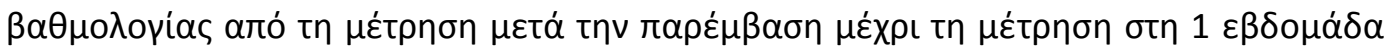

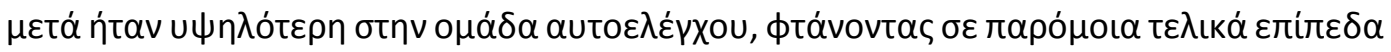
$\tau \iota \mu \dot{v}$.

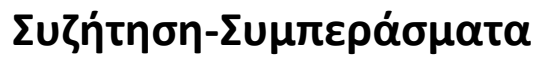

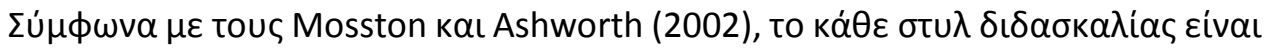

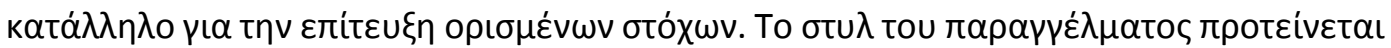

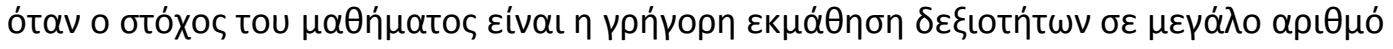

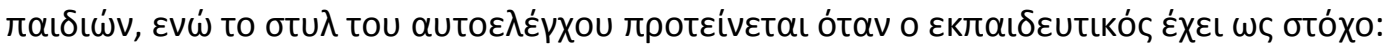

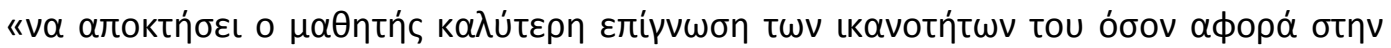

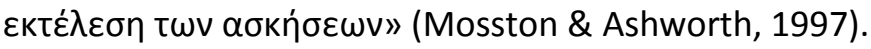

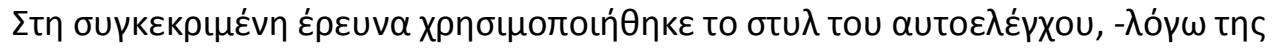

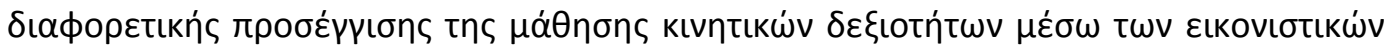

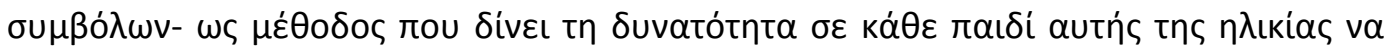

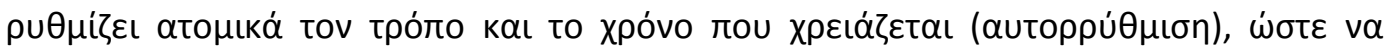

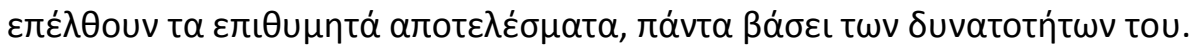

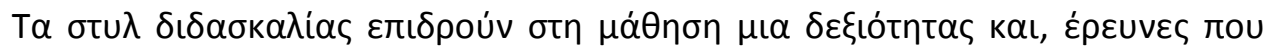

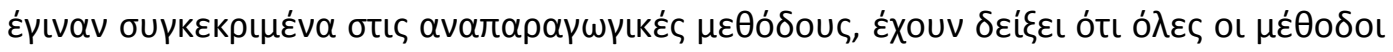

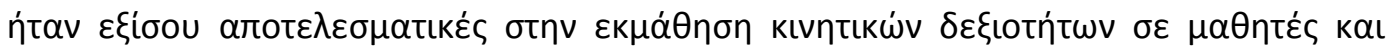

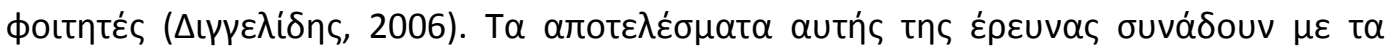

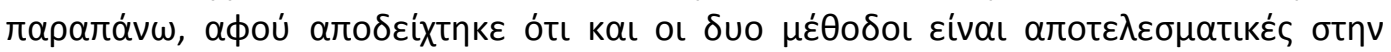

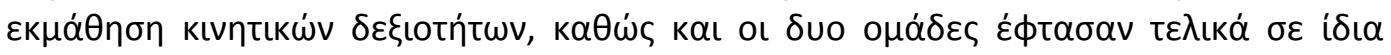

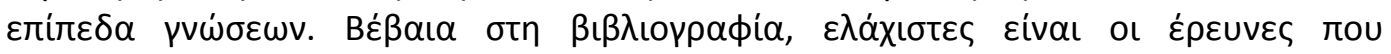

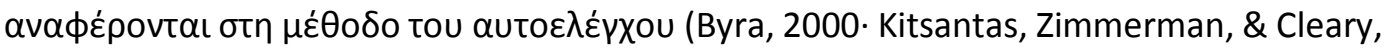

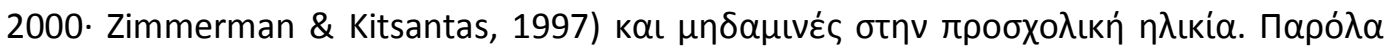

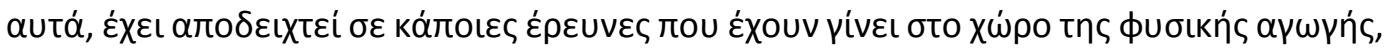

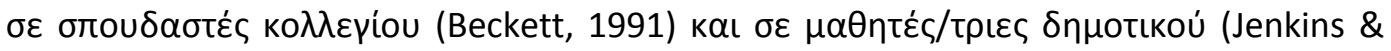

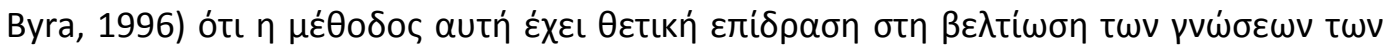

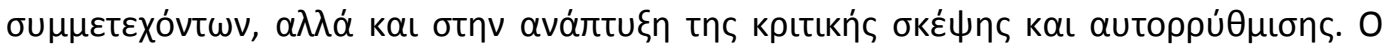

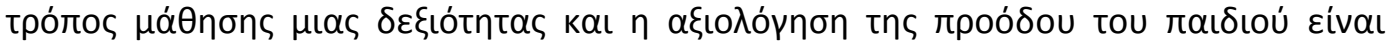

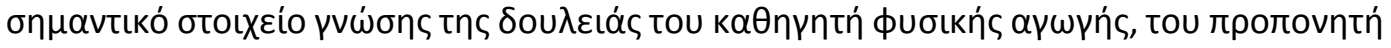

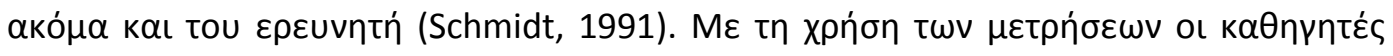

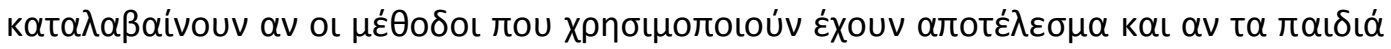

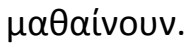

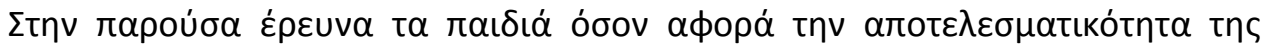

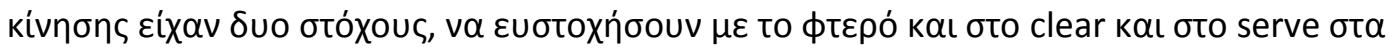

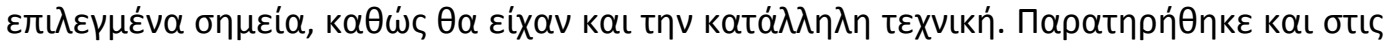

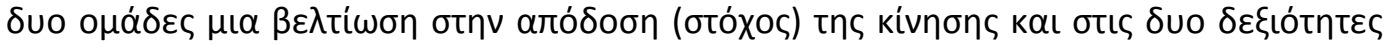

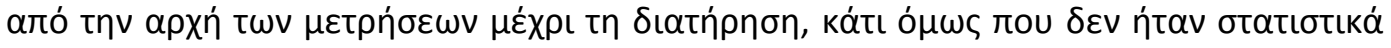

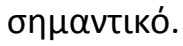




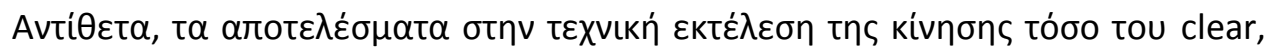

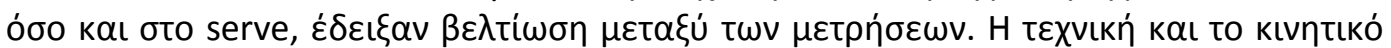

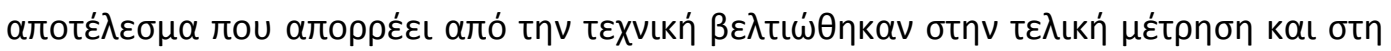

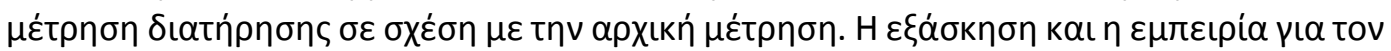

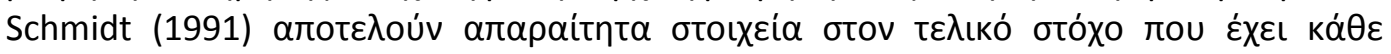

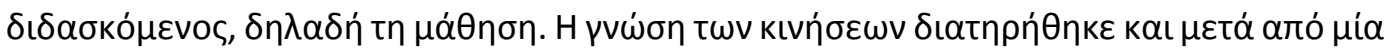

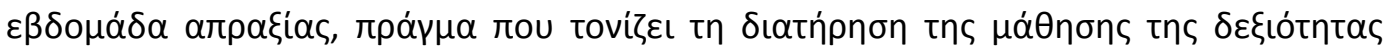

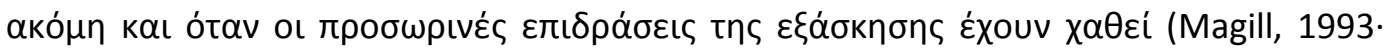

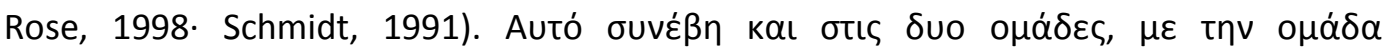

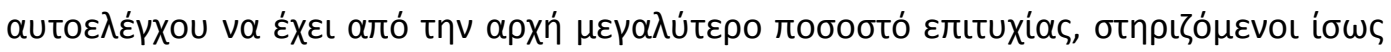

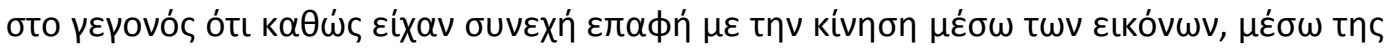

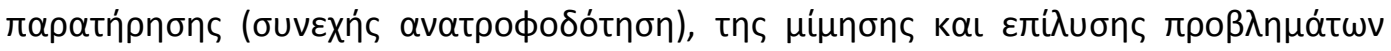

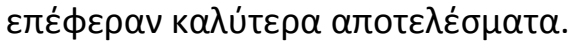

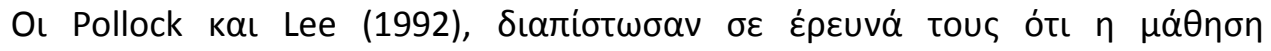

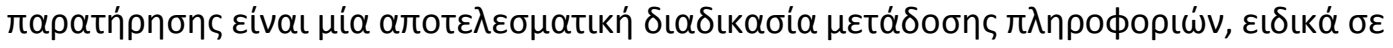

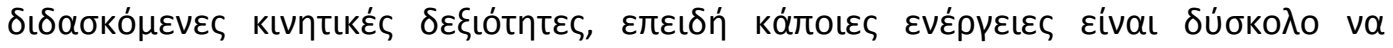

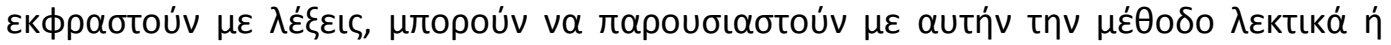

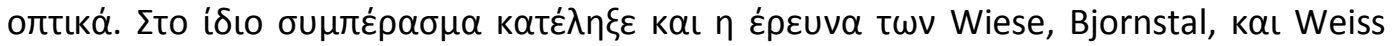

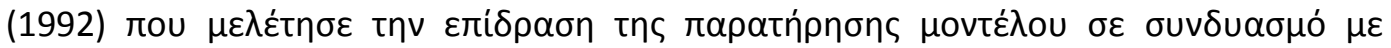

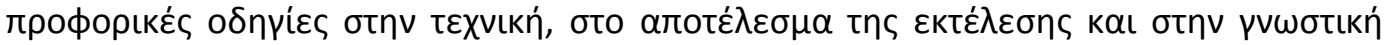

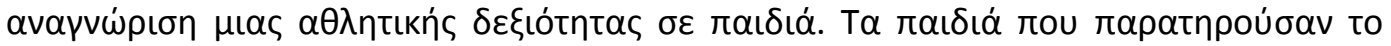

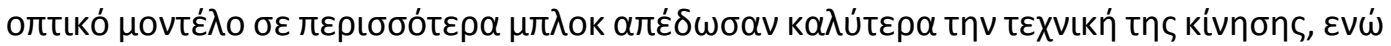

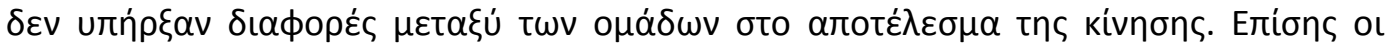

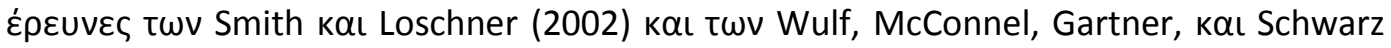

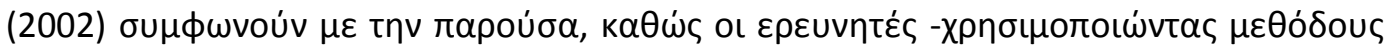

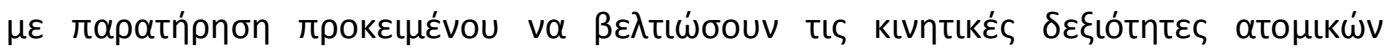

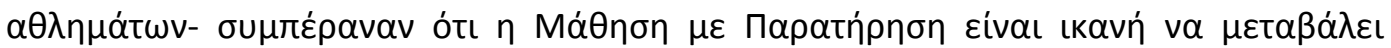

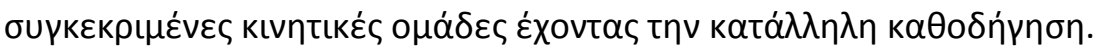

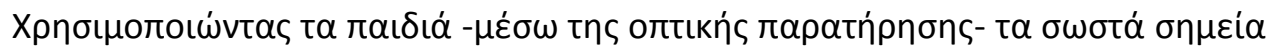

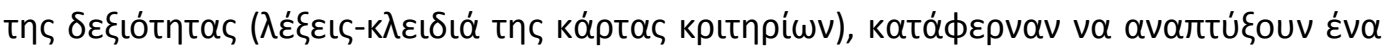

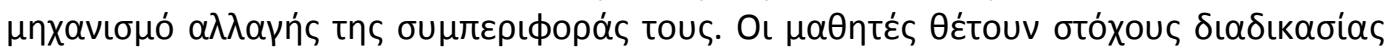

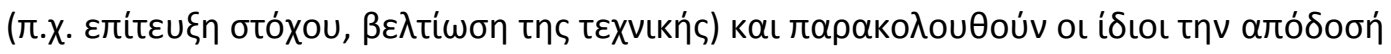

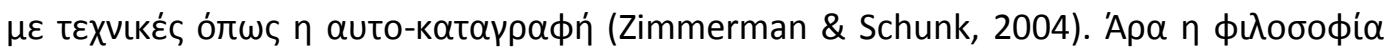

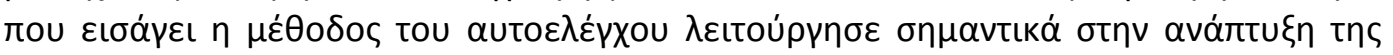

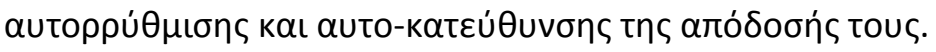

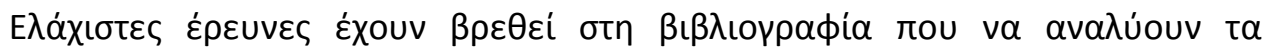

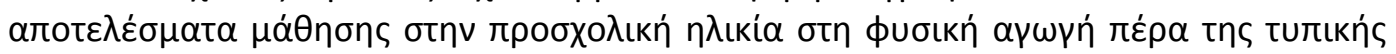

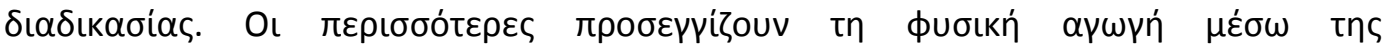

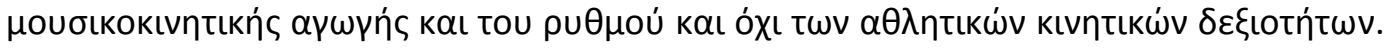

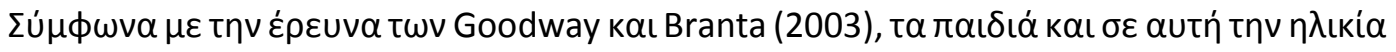

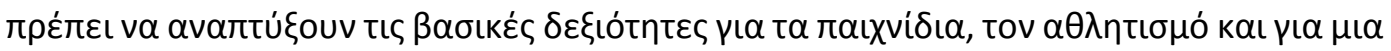

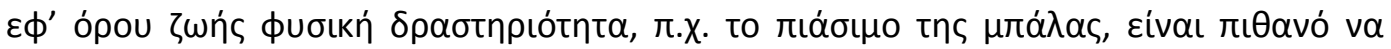

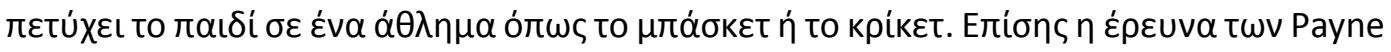

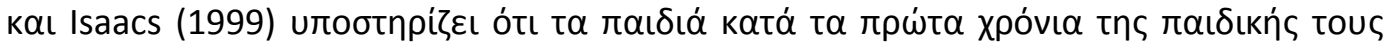

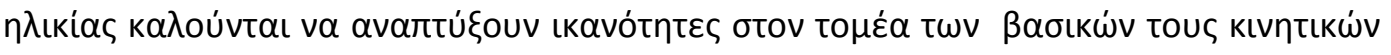
$\delta \varepsilon \xi$ เotńt $\omega v$. 


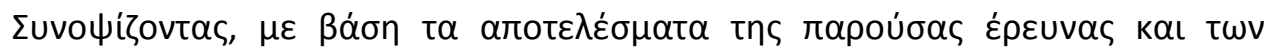

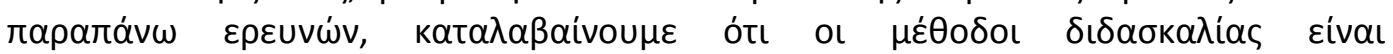

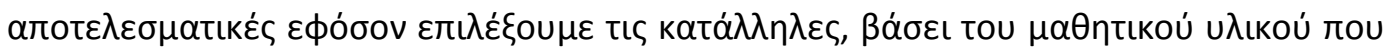

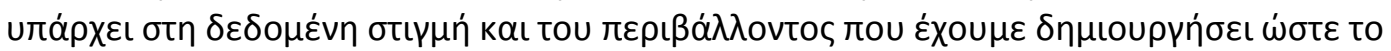

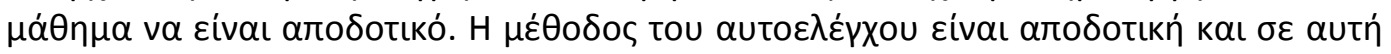

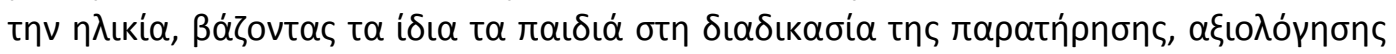

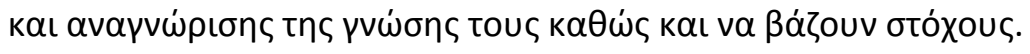

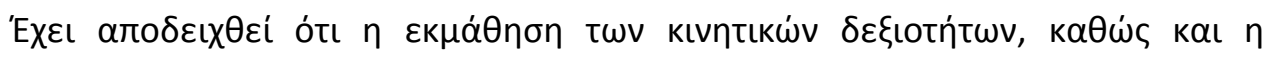

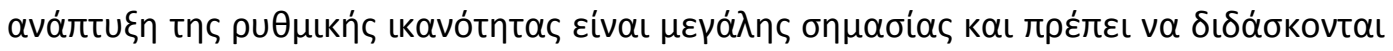

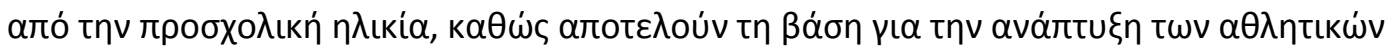

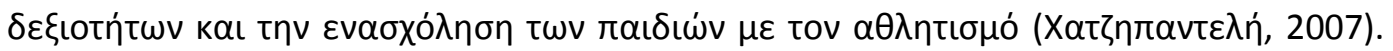

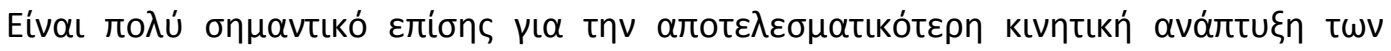

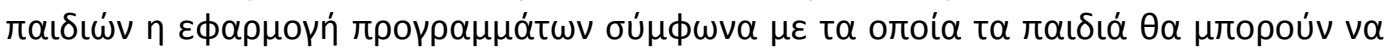

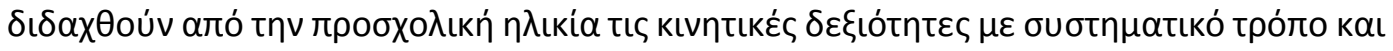

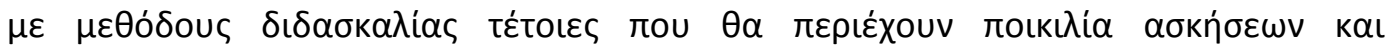

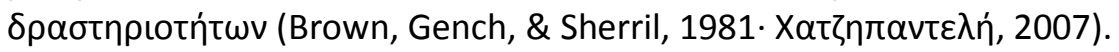

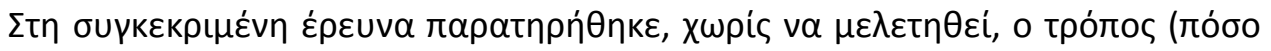

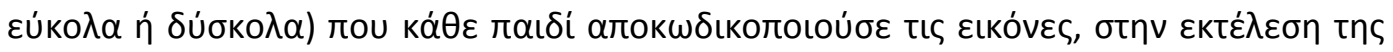

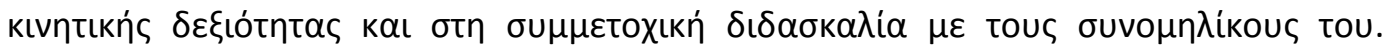

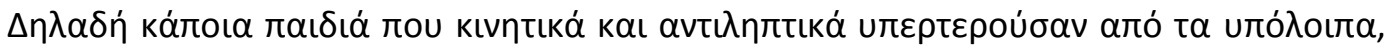

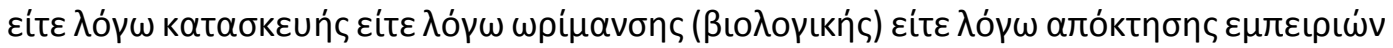

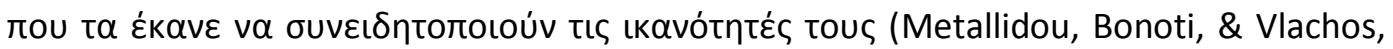

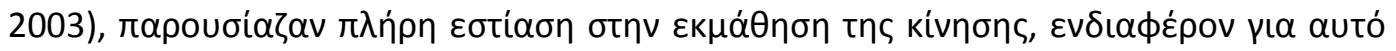

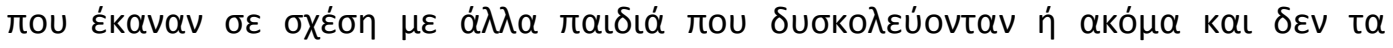

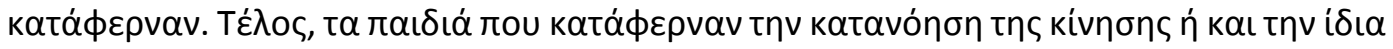

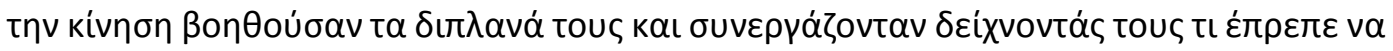
Kóvouv.

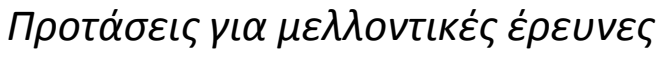

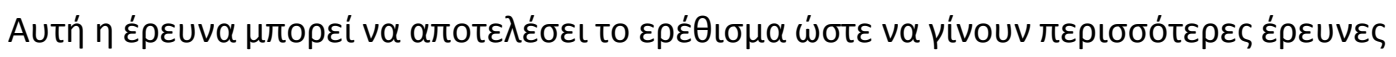

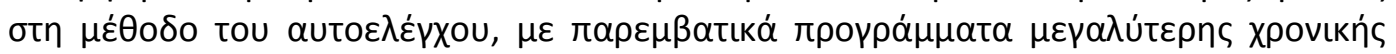

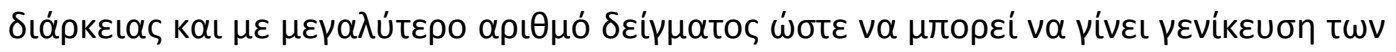

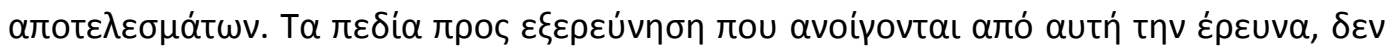

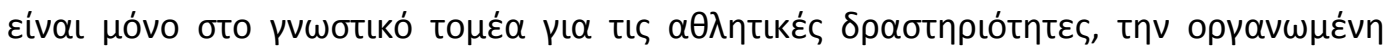

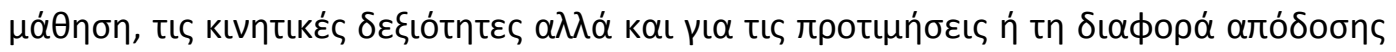

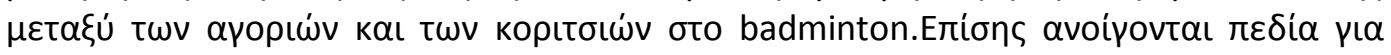

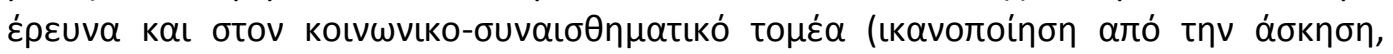

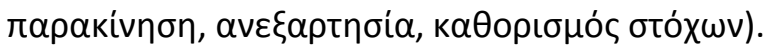

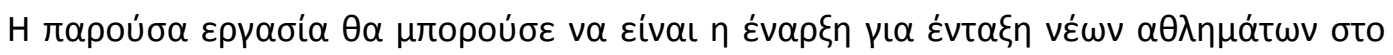

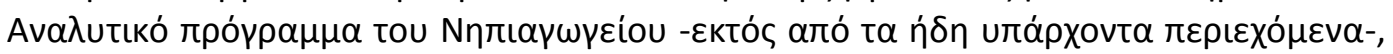

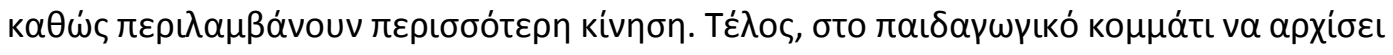

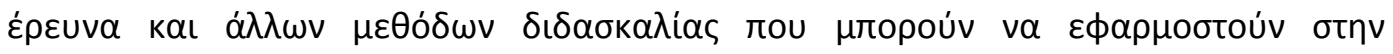

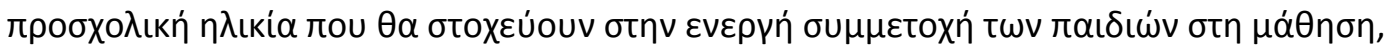

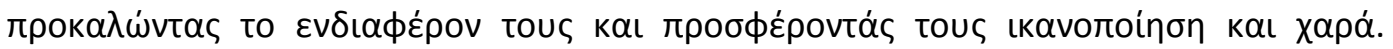

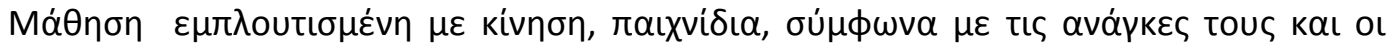

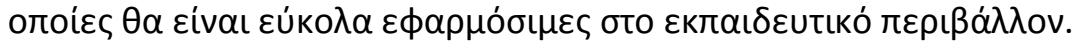




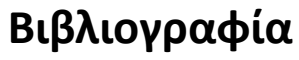

Avgerinou, M. (2009). Re-viewing visual literacy in the "Bain d' Images" Era. Tech Trends, 53(2), 28-34.

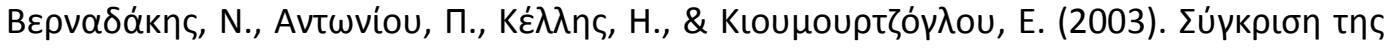

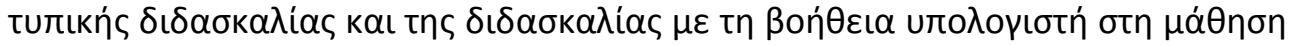

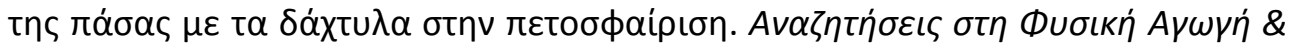

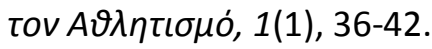

Badminton World Federation. (2011). Shuttle Time Schools Badminton Teachers Manual. Retrieved from www.bwfbadminton.org

Barrow, H., \& McGee, R. (1979). A practical approach to measurement in physical education. Philadelphia: Lea \& Febiger.

Beckett, K. (1990). The effects of two teaching styles on college students achievement of selected physical education outcomes. Journal of Teaching in Physical Education, 10, 153-169.

Begley, S. (1995). Do you hear what I hear. In E. N. Junn \& C. J. Boyatzis (Eds.), Child growth and development: Annual editions (pp. 44-48). London: Duskin Publishing Group.

Benassi, G. (2013). A "Shuttleton" handbook for MINI- BADMINTON. Italy. Retrieved from https://d3jc3ahdjad7x7.cloudfront.net/7BXjisuUiNTYZ

Brown, J., Sherril, C., \& Gench, B. (1981). Effects of an integrated physical education/music program in changing early childhood perceptual-motor performance. Perceptual and Motor Skills, 53, 151-154.

Byra, M. (2000). A review of spectrum research: The contribution of two eras'. Quest, 52, 22.

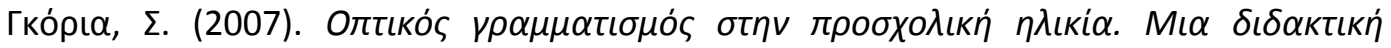

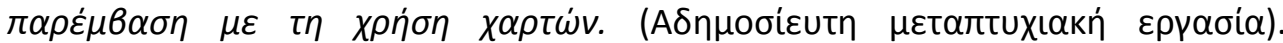

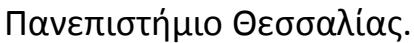

Campell, F. A., \& Ramey, C. T. (1994). Effects of early intervention on intellectual and academic achievement QA follow-up study of children from low-income families. Child Development, 65, 684-698.

Chu, L. I., \& Chen, W. C. (2000). Multimedia Application to Motor Skill Learning. In J. Bourdeau \& R. Heller (Eds.), Proceedings of Ed Media: World Conference on Educational Media and Technology (pp. 1257-1258). Montreal, USA: Association for the Advancement of Computing in Education (AACE).

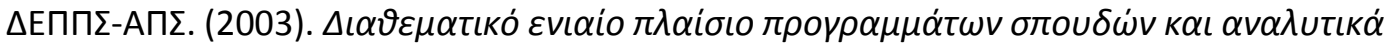

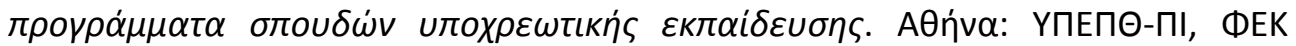

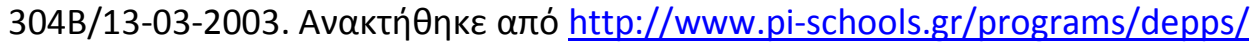

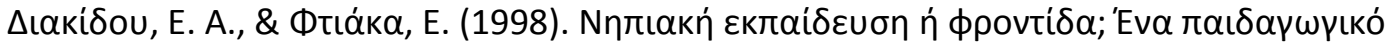

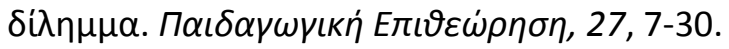




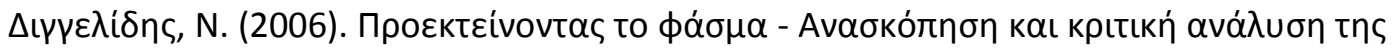

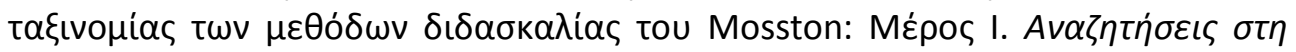

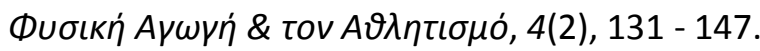

Debes, J. L. (1968). Some foundations for visual literacy. Audiovisual Instruction, 13, 96164.

DellaMattera, J. N. (2011). Perceptions of preservice early educators: How adults support preschoolers' social development. Journal of Early Childhood Teacher Education, 32, 26-38.

Dodge, D. T., \& Colker, L. J. (1998). The creative curriculum for early childhood. Washington DC: Teaching Strategies Inc.

Elliott, S. N., DiPerna, J. C., Mroch, A. A., \& Lang, S. C. (2004). Prevalence and patterns of academic enabling behaviors: An analysis of teachers' and students' ratings for a national sample of students. School Psychology Review, 33(2), 302-309.

Farrow, A. (1970). Skill and knowledge proficiencies for selected activities in the required program at Memphis State University. Retrieved from https://libres.uncg.edu/ir/uncg/f/Farrow uncg 7126936.pdf

French, E., \& Stalter, E. (1949). Study of Skill Tests in Badminton for College Women. The Research Quarterly, 32, 257-72.

Zimmerman, B. J., \& Kitsantas, A. (1997). Developmental phases in self-regulation: Shifting from process goals to outcome goals. Journal of Educational Psychology, 89, 29-36.

Zimmerman, B. J., \& Schunk, D. H. (2004). Self-regulating intellectual process and outcomes: A social cognitive perspective. In D. Y. Dai \& R. J. Sternberg (Eds.), Motivation, emotion and cognition (pp. 323-349). Mahwah, NJ: Lawrence Erlbau.

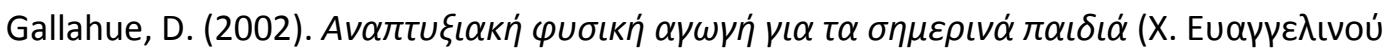

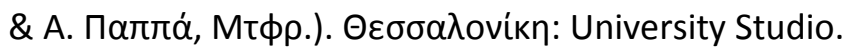

Gardner, H. (1991). The unschooled mind: How children think and how schools should teach. New York: Basic Books.

Goodway, J. D., \& Branta, C. F. (2003). Influence of a motor skill intervention on fundamental motor skill development of disadvantaged preschool children. Research Quarterly for Exercise and Sport, 74, 36-47.

Hellbrügge, T., Rutenfranz, A., \& Graf, O. (1966). Gesundheit und Leistungsfähigkeit im Kindes- und Jugendalter. Stuttgart: Kletta-Kott.

Ignico, A. (1997). The effects of interactive videotape instruction on knowledge performance and assessment of sport skills. Physical Educator, 54(2), 58-64.

Inkpen, K. M. (2001). Drag-and-drop versus point-and-click: mouse interaction styles for children. ACM Transactions on Computer Human Interaction, 8(1), 1-33.

Jenkins, J., \& Byra, M. (1996). An exploration of theoretical constructs associated with the spectrum of teaching styles. In F. Carreiro da Costa (Ed.), Research on teaching and research on teacher education: What do we know about the past and what kind of future do we expect? (pp. 103-108). Lisbon, Portugal: AIESEP. 
Johnson, R. (1995). The picture communication symbols guide, book I \& II. Solana Beach, CA: Mayer-Johnson, Inc.

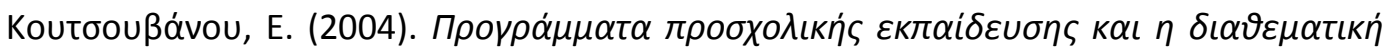

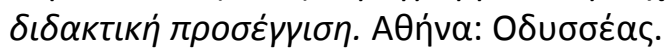

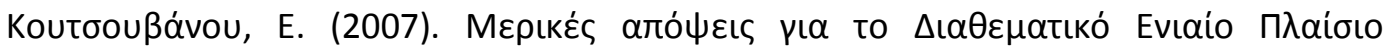

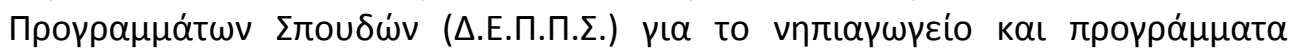

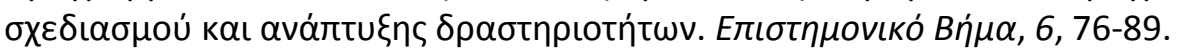

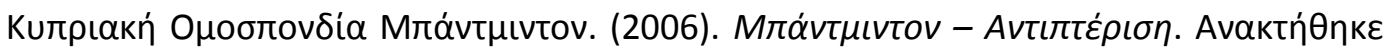
aró http://blogs.sch.gr/mkalfaki/files/2014/01/Badminton KOM.pdf

Kitsantas, A., Zimmerman, B. J., \& Cleary, T. (2000). The role of observation and emulation in the development of athletic self-regulation. Journal of Educational Psychology, 92, 811-817.

Levinson, D., \& Christensen, K. (1999). Encyclopedia of world sport from ancient times to the present. New York, Oxford: Oxford University Press.

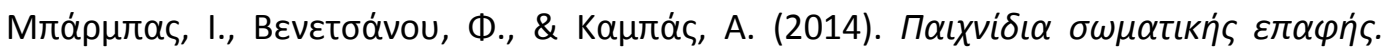
К $\alpha \beta \alpha ́ \lambda \alpha: \Sigma \alpha i ̈ t \alpha$.

Magill, R. A. (1993). Augmented feedback in skill acquisition. In R. N. Singer, M. Murphy, \& L. K. Tennant (Eds.), Handbook of research on sport psychology (pp. 193-212). New York: Macmillan.

Mayer, R. E., \& Moreno, R. (2003). Nine ways to reduce cognitive load in multimedia learning. Educational Psychologist, 38(1), 43-52.

Metallidou, P., Bonoti, F., \& Vlachos, F. (2003). Drawing performance, metacognitive experiences and handedness in school-age children. Scientific Annals of the Psychological Society of Northern Greece, 1, 205-229.

Mosston, M., \& Ashworth, S. (1994). Teaching physical education. Columbus, $\mathrm{OH}$ : Charles E. Merrill.

Mosston, M., \& Ashworth, S. (2002). Teaching physical education (5 th $^{\text {ed.). }}$. Boston: Benjamin Cummings.

Payne, V. G., \& Isaacs L. D. (1999). Human motor development. A lifespan approach ( $^{\text {th }}$ ed.). Maintain View, CA: Mayfield.

Pickens, J. (2009). Socio-emotional programme promotes positive behaviouring preschoolers. Child Carein Practice, 15(4), 261-278.

Pollock, B. J., \& Lee, T. D. (1992) Effects of the model's skill level on observational motor learning. Research Quarterly for Exercise and Sport, 63, 25-29.

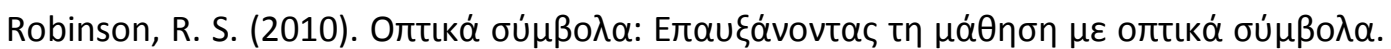

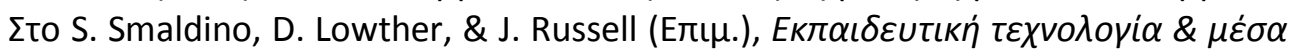

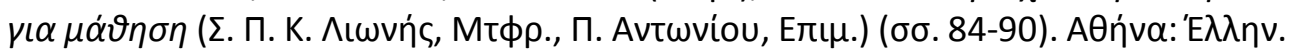

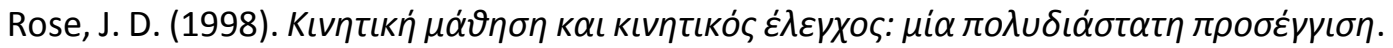

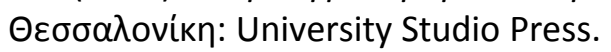


Rosenthal, M., \& Gatt, L. (2010). 'Learning to Live Together': training early childhood educators to promote socio-emotional competence of toddlers and pre-school children. European Early Childhood Education Research Journal, 18(3), 373-390.

Roth, K. \& Winter, K. (1994). Entwicklung koordinativer Fähigkeiten. In J. Baur, K. Boes, \& R. Singer (Eds.), Motorische Entwicklung. Ein Handbuch (pp. 191-216). Schorndorf: Hofmann.

Schmidt, R. A. (1991). Motor learning and performance. From principles to practice. Champaign, IL: Human Kinetics.

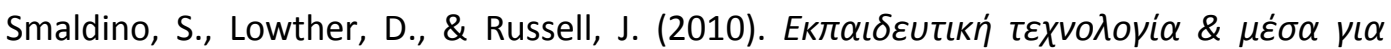

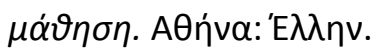

Smith, R. M., \& Loschner, C. (2002). Biomechanics feedback for rowing. Journal of Sports Sciences, 10, 783-791.

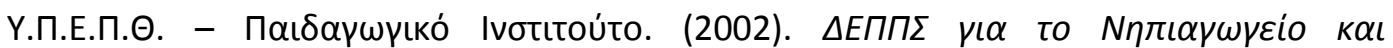

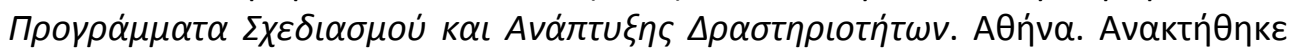
aró http://digitalschool.minedu.gov.gr

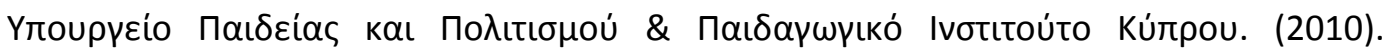

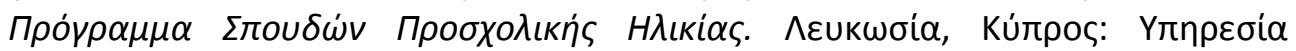

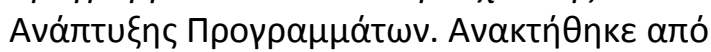
http://archeia.moec.gov.cy/mc/2/proscholiki ekpaidefsi.pdf

Yadav, S. K., \& Prajapati, G. (2011). Construction of knowledge test in badminton for physical education students. International Journal of Physical Education, Sports and Yogic Sciences, 1(1), 21-23.

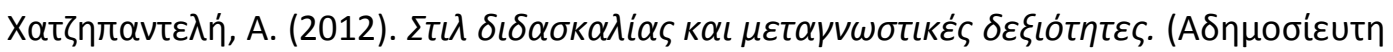

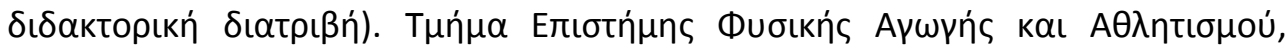

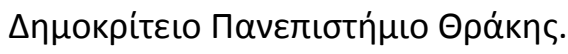

Wang, J. (2004). Developmental trends for badminton game play across skill levels: An exploratory study. (Unpublished doctoral dissertation). University of South Carolina, Columbia.

Wiese-Bjornstal, D. M., \& Weiss, M. R. (1992). Modeling effects on children's form kinematics, performance outcome, and cognitive recognition of a sport skill: An integrated perpective. Research Quarterly for Exercise and Sport, 63, 67-75.

Wulf, G., McConnel, N., Gartner, M., \& Schwarz, A. (2002). Enhancing the learning of sport skills through external-focus feedback. Journal of Motor Behavior, 34, 171-182. 